\title{
Correcting Distortion of Polarimetric SAR Data Induced by Ionospheric Scintillation
}

\author{
Jun Su Kim, Konstantinos P. Papathanassiou, Fellow, IEEE, Rolf Scheiber, and Shaun Quegan, Member, IEEE
}

\begin{abstract}
A correction methodology for distortions induced by ionospheric scintillation on fully polarimetric synthetic aperture radar (SAR) data is proposed. The correction is based on deriving the phase distortion induced by the ionosphere from Faraday rotation estimates. The estimated phase distortion is then used for correction. In order to compensate the phase and time-Doppler history distortions, the correction has to be performed at the slant range of the ionospheric layer, i.e., on partially focused single-look complex data. Accordingly, the performance of the proposed correction methodology depends, among other factors, on knowledge of the altitude of the effective ionospheric layer (assuming the thin ionospheric layer model). Its estimation from the SAR data itself is therefore also addressed. The methodology was applied and validated on simulated P-band data for various ionospheric conditions and on real L-band data acquired by the Advanced Land Observation Satellite Phased Array L-band SAR (PALSAR).
\end{abstract}

Index Terms-Faraday effect, ionosphere, polarimetry, spaceborne radar, synthetic aperture radar (SAR).

\section{INTRODUCTION}

$\mathbf{T}$ HE ionosphere is the upper part of the Earth's atmosphere where solar radiation ionizes gas molecules and atoms sufficiently to affect the propagation of radio waves [1]. Electron density significantly varies with altitude as a result of the competition between photochemical, collisional, and diffusion processes. A large fraction of the ionization is concentrated in a relatively narrow layer (known as the F2-layer [2, p. 1]) whose peak value is usually located at an altitude between 250 and $400 \mathrm{~km}$. For the purpose of propagation calculations, the ionosphere is often approximated by a thin layer whose spatial variation is characterized by the integrated value of the electron density along a given direction, known as the total electron content (TEC). TEC varies on spatial scales extending from a few meters to thousands of kilometers [3]. Small-scale ionospheric irregularities caused by particle precipitation and plasma instabilities induce scintillations [4] and are common features of the polar and post-sunset equatorial ionosphere [4]. Larger

Manuscript received August 1, 2014; revised December 23, 2014 and April 1, 2015; accepted April 22, 2015. This work was supported in part by the European Space Agency BIOMASS mission project.

J. S. Kim, K. P. Papathanassiou, and R. Scheiber are with the Microwaves and Radar Institute, German Aerospace Center (DLR-HR), Wessling 82234, Germany (e-mail: junsu.kim@dlr.de; Kostas.papathanassiou@dlr.de; rolf.scheiber@dlr.de).

S. Quegan is with the Centre for Terrestrial Carbon Dynamics, The University of Sheffield, Sheffield S3 7RH, U.K. (e-mail: S.Quegan@ @heffield.ac.kr).

Color versions of one or more of the figures in this paper are available online at http://ieeexplore.ieee.org.

Digital Object Identifier 10.1109/TGRS.2015.2431856 scale ionospheric features, which we will regard as constituting the background ionosphere, extend to global scale and are regulated by solar radiation and auroral precipitation, together with photochemical and transport processes [2]. Note that, in the context of synthetic aperture radar (SAR) data correction, the relevant scales of spatial variation in the ionosphere are with respect to the length of the synthetic aperture at the height of the ionospheric layer, which is usually on the order of several kilometers depending on radar frequency and imaging mode.

Due to the presence of free electrons in the ionosphere, pulses transmitted by the SAR and backscattered by a scatterer experience phase advance and group delay [5]. This can cause several types of distortion in the SAR image, including defocusing and/or spatial shifts in both range and azimuth [6], [7]. In addition, in the presence of the Earth's magnetic field, the ionosphere is anisotropic, and only circularly polarized waves propagate through it without changing their polarization (although at different velocities for the right and left circular waves). This effect is known as Faraday rotation (FR) [2], [8]-[10]. In the case of repeat-pass interferometric SAR, the individual images are distorted by the different ionospheric conditions at each acquisition time; if not accounted for, this difference induces a loss of interferometric coherence and/or a distortion of the interferometric phase [9], [11]-[13].

Ionospheric distortions become stronger as frequency decreases, so are more critical for low-frequency spaceborne SAR [5], [11] implementations. Nonetheless, a number of low-frequency spaceborne SAR missions operating at L- and P-bands are in space or planned to be launched in the next decade. These include the ESA BIOMASS mission for global forest biomass mapping, which is the first-ever spaceborne mission operating at P-band (435-MHz center frequency) in a fully polarimetric mode [14]. At L-band, Japan Aerospace Exploration Agency's (JAXA) Advanced Land Observation Satellite-2 (ALOS-2) mission [15] launched in 2014 and the first of the two SAOCOM (CONAE/ASI) SARs is expected to be placed in orbit in 2016 [16]. Other planned/proposed L-band SAR missions include the NISAR (NASA/ISRO) [17] and TanDEM-L of the German Aerospace Center (DLR) [18]. All of them will be, to some degree, affected by distortions induced by ionosphere irregularities; hence, appropriate mitigation approaches need to be developed. These can include selection of a dawn/dusk orbit that minimizes the effects [19], but correction may be still needed along some parts of the orbit, such as in the auroral zones.

In this paper, a methodology to correct amplitude and phase distortions of focused SAR images induced by scintillations in the azimuth direction is proposed. While gradients in the 
background ionosphere induce gradual spatial shifts [20]-[22], scintillations induce phase distortions [4], [23] that modify the time-Doppler history of the received pulses and, if left uncompensated, can cause defocusing and loss of contrast [3], [24], geometric distortions [6], and loss of interferometric coherence [12], [13], [21]. In the presence of point scatterers with sufficiently high signal-to-clutter ratio, the phase history of the scatterers can be directly evaluated to estimate the phase distortion [25]. In the absence of such scatterers, contrast optimization or multilook azimuth shift estimation techniques have been proposed. However, their performance strongly depends on the amplitude contrast within the images. In the following, an alternative methodology based on deriving the ionospheric phase distortion (in the following, also referred as ionospheric phase screen) from FR estimates is proposed. As our study focuses on azimuth defocusing and relies on FR estimates, high-latitude scintillation is emphasized.

In the equatorial post-sunset sector, the ionospheric irregularities often leave amplitude stripes on SAR image [26]. They are caused by the diffraction [27] of the SAR pulses through the irregularities aligned approximately parallel to the sunsynchronous SAR orbit and almost parallel to the geomagnetic field, which exerts a strong control on the direction of equatorial ionospheric irregularities. Moreover, because of the small FR around the equator, the equatorial ionospheric effects are left outside of the scope of this paper. The range scintillations are not considered: The spatial variation of the ionospheric irregularity can hardly induce multipath effects [11], and the short pulse duration (on the order of microseconds) makes scintillation hardly possible because the temporal evolution of the ionosphereic irregularities is negligible.

The effect of the ionosphere on microwave propagation and the relations between TEC, FR, and ionospheric phase distortion are reviewed in Section II. This provides the basis for the discussion of the effects of ionospheric irregularities on azimuth focusing in Section III. In Section IV, existing FR estimators are reviewed, and their estimation accuracy is discussed. The scintillation correction algorithm is described in Section V, and Section VI deals with testing its performance by means of simulated P-band data under various ionospheric conditions and with real L-band data acquired by the ALOS Phased Array L-band SAR (PALSAR). This paper is concluded in Section VII. Finally, a new approach using the SAR data itself to estimate the ionospheric altitude is set out in the Appendix.

\section{IONOSPhERIC EFFECTS ON MicrowaVe Propagation}

The index of refraction $n$ for microwaves propagating in the Earth's ionosphere is given by the Appleton-Hartree equation [28]. Its approximation for high frequencies and nonabsorptive media simplifies to

$$
n_{\mp}=1-\frac{1}{2} \frac{f_{p}^{2}}{f^{2}}\left(1 \mp \frac{f_{H}}{f}\right)
$$

where $f_{p}^{2}=N e^{2} / 4 \pi^{2} \epsilon_{0} m_{e}$ is the square of the plasma frequency $f_{p}, f_{H}=e \vec{B} \cdot \hat{\kappa} /\left(2 \pi m_{e}\right)$ is the electron cyclotron frequency, and $f$ is the carrier frequency. In these expressions, $N$ is the electron density, $\vec{B}$ is the geomagnetic field, $\hat{\kappa}=\vec{\kappa} /|\vec{\kappa}|$ is the unit wave propagation vector, $e$ and $m_{e}$ are the charge and the mass of the electron, and $\epsilon_{0}$ is the free-space permittivity. The $\mp$ subscript is related to the birefringence of the ionosphere, which is discussed in Section II-B. The propagation vector $\vec{\kappa}$ is related to the frequency since $|\vec{k}|=2 \pi c_{0} / f$ in free space, where $c_{0}$ is the speed of light. The refractive, dispersive, and anisotropic characteristics of the Earth's ionosphere are determined by (1).

\section{A. Phase Advance}

According to (1), the phase velocity of microwaves is larger in the ionosphere than in free space as $n$ is always smaller than one. Hence, the phase of the pulses received by the SAR through the ionosphere is advanced relative to the ionospherefree case. Integrating along the propagation path twice through the ionosphere $C(l)$, where $l$ is the propagation length, the phase advance of round trip $\phi$, as compared to free-space propagation, is given by

$$
\phi=2 \cdot 2 \pi f \int_{C(l)} \frac{n-1}{c_{0}} d l .
$$

Since $f_{H} / f$ is small, substituting (1) into (2) yields the good approximation

$$
\phi=4 \pi \zeta \frac{T E C}{c_{0} f}
$$

where

$$
\zeta=\frac{e^{2}}{8 \pi^{2} \epsilon_{0} m_{e}}=\frac{e^{2} c_{0}^{2}}{2 \pi m_{e}} \times 10^{-7}=40.3082 \mathrm{~m}^{3} / \mathrm{s}^{2}
$$

and $T E C=\int_{C} N d l$. Equation (3) indicates the high sensitivity of phase advance to TEC [9], [12], [13]. For example, 1 TEC unit (TECU) $\left(=1.0 \times 10^{16}\right.$ electrons $\left./ \mathrm{m}^{2}\right)$ induces $13.3 \mathrm{rad}$ of phase advance at L-band $(f=1.27 \mathrm{GHz})$ and $38.9 \mathrm{rad}$ at P-band $(f=435 \mathrm{MHz})$. Even at higher frequencies such as X-band $(f=9.35 \mathrm{GHz}), 1$ TECU induces a phase advance of $1.81 \mathrm{rad}$.

Usually, TEC is defined along a vertical path, i.e., $T E C_{V} \equiv$ $\int_{r} N d r$, where $r$ runs radially from the Earth's surface to the altitude where the measurements are performed. For example, the IONEX archives [29] built up from the Global Positioning System provide TEC estimates with $r$ in the range of 0-20 $200 \mathrm{~km}$. For spaceborne SAR, TEC is defined along the propagation path from the SAR sensor to the given resolution cell located on the ground. Using the thin layer approximation, $T E C:=T E C_{V} / \cos \theta$, where $\theta$ is the incidence angle at the effective altitude of the thin ionospheric layer.

\section{B. $F R$}

Birefringence in the ionosphere gives rise to two refractive indices, indicated by the $\mp$ sign in (1), each of which has a corresponding characteristic wave, or eigenwave, whose polarimetric state does not change during propagation through 


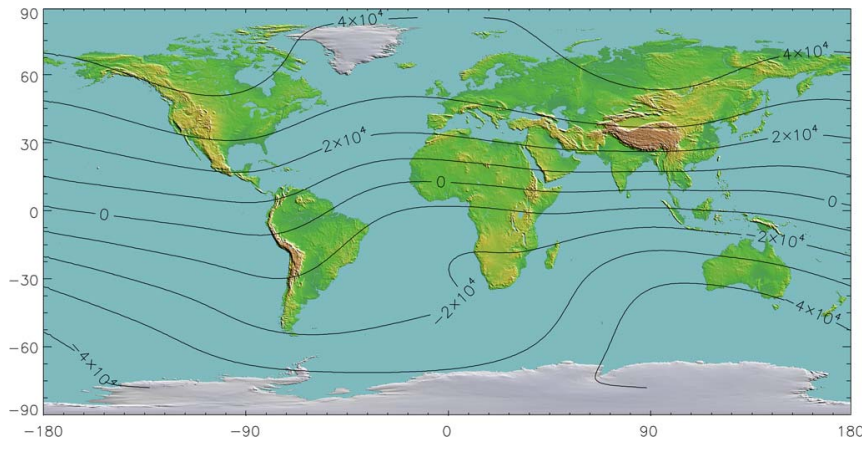

Fig. 1. Global map of $\vec{B} \cdot \hat{\kappa}$ at $400-\mathrm{km}$ altitude in units of nanoteslas for a sun-synchronous orbit of right-looking SAR with $30^{\circ}$ off-nadir angle.

the ionosphere. The difference between these refractive indices is

$$
\Delta n=n_{+}-n_{-}=\frac{N \zeta e \vec{B} \cdot \hat{\kappa}}{\pi m_{e} f^{3}} .
$$

The corresponding phase difference after one-way propagation is given by

$$
2 \Omega=\frac{2 \pi f}{c_{0}} \int_{C} \Delta n d l
$$

where $\Omega$ is called the one-way FR angle. When the plane of polarization rotates by $\Omega$ around $\vec{\kappa}$, the phase of the $n_{+}$ characteristic wave is $2 m \pi+\Omega$, whereas that of the $n_{-}$wave is $2 m \pi-\Omega$, for some integer $m$. This explains the factor 2 appearing in (6). Treating $\vec{B} \cdot \hat{\kappa}$ as a constant along the integration path $C$ yields the relation between FR and TEC

$$
\Omega=\frac{\zeta(e \vec{B} \cdot \hat{\kappa})}{c_{0} m_{e} f^{2}} T E C .
$$

From (6), it follows that an accurate FR estimate obtained from $\int_{C}(\vec{B} \cdot \hat{\kappa}) N d l$ requires knowledge of both the electron density and the geomagnetic field $\vec{B}$ along the integration path $C$. As function $\vec{B} \cdot \hat{\kappa}$ is continuous and $N(l)$ is always positive, $\min (\vec{B} \cdot \hat{\kappa}) T E C \leq \int_{C} \vec{B}(l) \cdot \hat{\kappa} N(l) d l \leq$ $\max (\vec{B} \cdot \hat{\kappa}) T E C$. In addition, because $\vec{B} \cdot \hat{\kappa}$ is monotonic in the integration interval, its extremes always appear at both ends of $C(l)$. When the FR refers to a simplified thin ionospheric layer at the altitude of $h_{\text {iono }}$, the magnetic field also corresponds to $h_{\text {iono }}\left(=\vec{B}_{\text {iono }}\right)$. According to the intermediate value theorem, there exists an $h_{\text {iono }}$ in the interval $\left[0, h_{\text {sat }}\right]$ that satisfies $\left(\vec{B}_{\text {iono }} \cdot \hat{\kappa}\right) \int_{C} N d l=\int_{C} \vec{B}(l) \cdot \hat{\kappa} N(l) d l$. For the remainder of this paper, the magnetic field is understood to have the value $\vec{B}_{\text {iono }}$ at this representative ionospheric altitude and is written simply as $\vec{B}$ for the sake of brevity.

The value of $\vec{B} \cdot \hat{\kappa}$ depends also on the orbit and the imaging geometry, which determine $\hat{\kappa}$. The global distribution of $\vec{B} \cdot \hat{\kappa}$ estimated at $400-\mathrm{km}$ altitude for a sun-synchronous orbit with $98^{\circ}$ inclination and a right-looking SAR geometry with offnadir angle of $30^{\circ}$ is shown in Fig. 1. The Earth's magnetic field is calculated from the US/UK World Magnetic Model for 2005-2010 [30]. The absolute value of $\vec{B} \cdot \hat{\kappa}$ increases toward

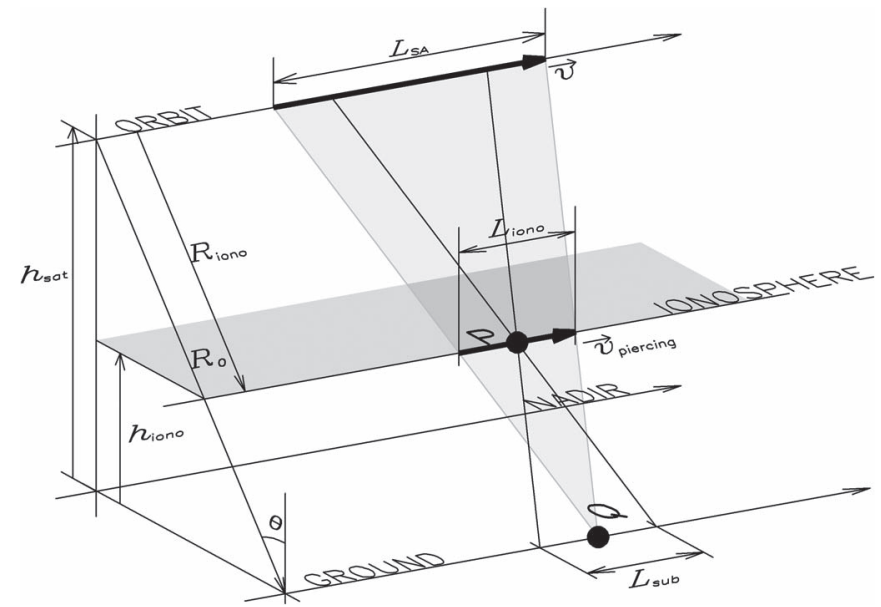

Fig. 2. SAR imaging geometry through the ionosphere.

the polar regions, but with opposite signs in each hemisphere, and becomes zero near the geomagnetic equator. As its global distribution is highly correlated with the geomagnetic latitude, the parallel geomagnetic field $\vec{B} \cdot \hat{\kappa}$ in units of nanoteslas will be used as a proxy for the geomagnetic latitude.

It is important to clarify that, in this paper, the ionospheric phase advance is defined for two-way propagation, and the FR angle is defined as one-way propagation. This is due to the characteristics of the ionospheric phase advance and the FR. The ionospheric phase advance is directly measureable from interferometry, and it is essentially a round-trip measure. In contrast, the estimation of FR in polarimetric SAR is based on one-way propagation (see Section IV). Despite the confusions at first glance, it facilitates the applications to actual SAR data because they are data-oriented definitions and are both functions of TEC.

\section{IONOSPHERIC EFFECTS ON AZIMUTH FoCUSING}

\section{A. SAR Imaging Geometry Through the Ionosphere}

Fig. 2 shows the geometry of a spaceborne SAR at altitude $h_{\text {sat }}$ imaging through a thin ionospheric layer at altitude $h_{\text {iono. }}$. A scatterer on the ground is imaged along the synthetic aperture $L_{\mathrm{SA}}$, and the point where the line connecting the sensor and the scatterer intersects the ionospheric layer is called the piercing point. For a sensor with velocity $v$, the piercing point moves with velocity $v_{\text {piercing }}=v \cdot\left(h_{\text {iono }} / h_{\text {sat }}\right)$. The phase history of a point $Q$ on the ground is superimposed on the phase disturbances induced by the ionosphere along $L_{\text {iono }}$. The phase disturbance at a point $P$ located at the ionospheric layer spreads due to the focusing over all scatterers within the length $L_{\text {sub }}$ in Fig. 2. Subscript "sub" stands for "sub-ionospheric point" or "the length subjected to the effect at a point of the thin ionospheric layer."

Accordingly, the TEC map should not be calculated from and/or applied on the focused single-look complex (SLC) images directly. Instead, the SLC images and the TEC maps are made "compatible" by "de"-compressing the SLC data to the 
altitude of the ionosphere, i.e., $h_{\text {iono }}$. This will be discussed in Section $\mathrm{V}$ and in the Appendix separately.

It is noteworthy that $L_{\mathrm{sub}}=L_{\text {iono. }}$. The ionospheric phase in $L_{\text {iono }}$ distorts a point $Q$ in the focused SAR data in the same way as the scattering phases in $L_{\text {sub }}$ distort the ionospheric phase estimation at a point $P$ in the defocused SAR data.

\section{B. Azimuth Shift}

The position along azimuth at which a given scatterer is imaged is determined by the time-Doppler relation. The ideal time-Doppler relation without the ionosphere is

$$
\frac{f_{a}(t)}{f}=\frac{2 v_{\mathrm{rel}}}{c_{0}}=\frac{2 v^{2} t}{R_{0} c_{0}}=\frac{D_{f}}{f} t
$$

where $f_{a}$ is the Doppler frequency, $v_{\text {rel }}$ is the relative velocity between the sensor and the scatterer, and $D_{f}$ is the Doppler rate. A linear TEC gradient along azimuth introduces a linear ionospheric phase component in the time-Doppler relation

$$
\frac{f_{a}(t)}{f}=\frac{1}{2 \pi f} \frac{\partial \phi}{\partial t}=-\frac{2 \zeta}{c_{0} f^{2}} \frac{\partial T E C}{\partial t}=-2 \zeta \frac{v_{\text {piercing }}}{c_{0} f^{2}} \frac{\partial T E C}{\partial x}
$$

where the time derivative of (3) is divided by the center frequency, and $(\partial T E C / \partial t)=(\partial T E C / \partial x) \cdot(d x / d t)$ is applied. Note that $(\partial x / \partial t)=v_{\text {piercing, }}$, not $v$. The negative sign in the last term represents the phase advance defined in (3). Assuming zero-squint geometry, a scatterer is focused at the azimuth time when the Doppler frequency becomes zero. When imaged through an ionosphere with a linear TEC gradient, it will be focused at a shifted position given by

$$
\Delta a=2 \zeta v \frac{v_{\text {piercing }}}{c_{0} f D_{f}} \frac{\partial T E C}{\partial x} .
$$

In interferometric applications, if the azimuth TEC gradient $\partial T E C / \partial x$ is different in the master and the slave acquisitions, the focusing positions of the scatterers are no longer identical, leading, if not compensated, to a loss of coherence in the interferogram [12], [13].

\section{TEC Irregularities and Scintillation}

The spatial scale and amplitude of TEC irregularities are often found to follow a power law scale-size spectrum [31]. The degree of scintillation is measured in terms of the power spectral density of TEC $\left(\left\langle(T E C-\overline{T E C})^{2}\right\rangle\right)$ at the wavenumber of $1 \mathrm{~km}^{-1}$, or $C_{k} L$, where $\overline{T E C}$ represents the spatial average of TEC [32]. Because of the large dynamic range of $C_{k} L$ (about five orders of magnitude) [3], it is more practical to use its confidence interval (CI) expressed as a percentile instead of its absolute (logarithmic) value. For example, $C_{k} L \mathrm{CI}=50 \%$ means that the instantaneous $C_{k} L$ is lower than the given value for $50 \%$ of time under given geophysical conditions, e.g., planetary number $\mathrm{Kp}$ (an index of the disturbance of the geomagnetic field), the location on the Earth, the time of day and year, and the phase in the solar cycle. Larger CI values indicate stronger but rarer scintillation conditions.

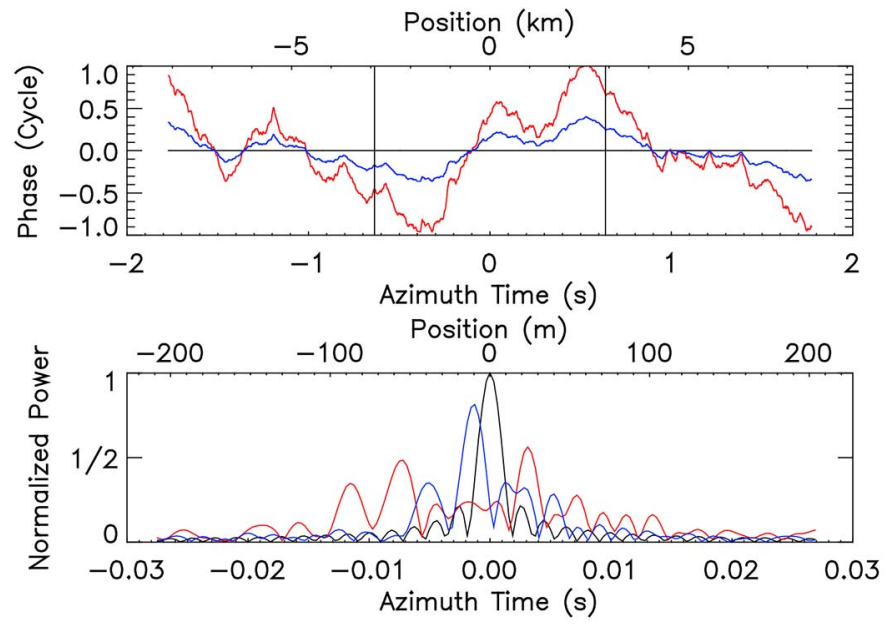

Fig. 3. Effect of ionospheric phase screen on azimuth focusing. The plot on the top shows the small-scale ionospheric irregularities converted to ionospheric phases using (3). The black, blue, and red lines indicate scintillation-free, weakscintillation, and strong-scintillation cases, respectively. The bottom plot shows the impulse response for the three cases.

Fig. 3 illustrates the effect of scintillation on the azimuth impulse response. The top figure shows the azimuth profiles of the small-scale ionospheric irregularities converted to ionospheric phases using (3). The black, blue, and red profiles are for zero, weak $\left(\mathrm{Kp}=1, C_{k} L \mathrm{CI}=90 \%\right)$ and strong $(\mathrm{Kp}=7$, $\left.C_{k} L \mathrm{CI}=90 \%\right)$ scintillation conditions, respectively. The two vertical lines indicate the synthetic aperture corresponding to the BIOMASS P-band radar assuming a $12.5-\mathrm{m}$ azimuth resolution [14]. The SAR system parameters and the geophysical parameters of these $C_{k} L$ statistics are described in detail in Section VI-A.2. The bottom figure shows the corresponding impulse responses for zero (black), weak (blue), and strong (red) scintillation conditions. Reduction of the peak sidelobe ratio occurs for weak scintillation conditions, but for strong scintillations, the impulse response is completely deformed. Moreover, the blue profile exhibits a shift corresponding to a gently increasing linear trend in the ionospheric phase (top figure) within the synthetic aperture. The effects of various degrees of scintillation on P-band and L-band SAR data were extensively studied in recent publications [19], [33].

\section{ESTIMATION OF FR}

\section{A. Scattering Matrix and Covariance Matrix Under FR}

In the presence of FR and assuming that system effects (crosstalk, channel imbalance, and noise) are negligible or have been corrected, the measured scattering matrix $\mathbf{O}=$ $\left(\begin{array}{ll}O_{h h} & O_{h v} \\ O_{v h} & O_{v v}\end{array}\right)$ is given by [34, p. 205], [35]

$$
\mathbf{O}=e^{i \phi_{\text {iono }}} \mathbf{R S R}
$$

where $\mathbf{S}=\left(\begin{array}{ll}S_{h h} & S_{h v} \\ S_{v h} & S_{v v}\end{array}\right)$ is the underlying scattering matrix, $\mathbf{R}=\left(\begin{array}{cc}\cos \Omega & \sin \Omega \\ -\sin \Omega & \cos \Omega\end{array}\right)$ represents the rotation of the polarization plane by the one-way FR angle $\Omega$, and $\phi_{\text {iono }}$ is the 
ionospheric phase advance defined in (3) [9]. Applying scattering reciprocity $\left(S_{h v}=S_{v h}=S_{x x}\right)$, (11) can be expanded to give

$$
\begin{aligned}
O_{h h} & =e^{i \phi_{\text {iono }}}\left(S_{h h} \cos ^{2} \Omega-S_{v v} \sin ^{2} \Omega\right) \\
O_{h v} & =e^{i \phi_{\text {iono }}}\left(S_{x x}+\left(S_{h h}+S_{v v}\right) \sin \Omega \cos \Omega\right) \\
O_{v h} & =e^{i \phi_{\text {iono }}}\left(S_{x x}-\left(S_{h h}+S_{v v}\right) \sin \Omega \cos \Omega\right) \\
O_{v v} & =e^{i \phi_{\text {iono }}}\left(S_{v v} \cos ^{2} \Omega-S_{h h} \sin ^{2} \Omega\right) .
\end{aligned}
$$

It is clear that one consequence of FR is the loss of scattering reciprocity; thus, $O_{h v} \neq O_{v h}$. The common phase component $e^{i \phi_{\text {iono }} \text { is neglected in the following. }}$

The covariance matrix $\mathbf{C}$ under FR is defined as

$$
\mathbf{C}=\left\langle\vec{k} \cdot \vec{k}^{* T}\right\rangle=\left(\begin{array}{cccc}
C_{11} & C_{12} & C_{13} & C_{14} \\
C_{12}^{*} & C_{22} & C_{23} & C_{24} \\
C_{13}^{*} & C_{23}^{*} & C_{33} & C_{34} \\
C_{14}^{*} & C_{24}^{*} & C_{34}^{*} & C_{44}
\end{array}\right)
$$

where $\vec{k}=\left(\begin{array}{llll}O_{h h} & O_{h v} & O_{v h} & O_{v v}\end{array}\right)^{T}$. Then, using (12) and assuming reflection symmetry for natural distributed scatterers, i.e., $\left\langle S_{h h} S_{x x}^{*}\right\rangle=\left\langle S_{x x} S_{v v}^{*}\right\rangle=0$, the diagonal elements of $\mathbf{C}$ are given by

$$
\begin{aligned}
& C_{11}=c^{2} \rho_{H H}-2 c s \Re\left\{\rho_{H V}\right\}+s^{2} \rho_{V V} \\
& C_{22}=x^{2}\left(\rho_{H H}+2 \Re\left\{\rho_{H V}\right\}+\rho_{V V}\right) \rho_{X X} \\
& C_{33}=x^{2}\left(\rho_{H H}+2 \Re\left\{\rho_{H V}\right\}+\rho_{V V}\right) \rho_{X X} \\
& C_{44}=s^{2} \rho_{H H}-2 c s \Re\left\{\rho_{H V}\right\}+c^{2} \rho_{V V}
\end{aligned}
$$

where $\Re$ denotes the real part, and the off-diagonal elements are given by

$$
\begin{aligned}
& C_{12}=c x \rho_{H H}+x\left(c \rho_{H V}-s \rho_{H V}^{*}\right)-s x \rho_{V V} \\
& C_{13}=-c x \rho_{H H}-x\left(c \rho_{H V}-s \rho_{H V}^{*}\right)+s x \rho_{V V} \\
& C_{14}=-c s \rho_{H H}+c^{2} \rho_{H V}-s^{2} \rho_{H V}^{*}-c s \rho_{V V} \\
& C_{23}=-x^{2}\left(\rho_{H H}+2 \Re\left\{\rho_{H V}\right\}+\rho_{V V}\right)+\rho_{X X} \\
& C_{24}=-s x \rho_{H H}+x\left(c \rho_{H V}-s \rho_{H V}^{*}\right)+c x \rho_{V V} \\
& C_{34}=s x \rho_{H H}-x\left(c \rho_{H V}-s \rho_{H V}^{*}\right)-c x \rho_{V V}
\end{aligned}
$$

with $c \equiv \cos ^{2} \Omega, s \equiv \sin ^{2} \Omega, x \equiv \sin \Omega \cos \Omega$, and $\rho_{p p} \equiv$ $\left\langle S_{p p} S_{p p}^{*}\right\rangle$ for $p=H, V$, or $X$, and $\rho_{H V} \equiv\left\langle S_{h h} S_{v v}^{*}\right\rangle$.

FR can be estimated from either the measured scattering matrix (12) or the measured covariance matrix (14) and (15) [36]. The most frequently used scattering-matrix-based estimators are the Bickel and Bates estimator [37] and Freeman's "first" estimator [38]. Important covariance-matrix-based estimators include Freeman's "second" estimator [38] and the Chen and Quegan estimator [39].

\section{B. FR Estimators}

1) Bickel and Bates Estimator: The Bickel and Bates estimator of $\Omega$ uses the phase difference between the crosspolarized channels of the observed scattering matrix expressed in a circular basis $\mathbf{O}_{C}[34$, p. 56]

$$
\mathbf{O}_{C}=\left(\begin{array}{ll}
O_{11} & O_{12} \\
O_{21} & O_{22}
\end{array}\right)=\frac{1}{2}\left(\begin{array}{cc}
-i & 1 \\
1 & -i
\end{array}\right)\left(\begin{array}{cc}
O_{h h} & O_{h v} \\
O_{v h} & O_{v v}
\end{array}\right)\left(\begin{array}{ll}
1 & i \\
i & 1
\end{array}\right) .
$$

Hence

$$
\begin{aligned}
O_{11} & =\frac{-i O_{h h}+O_{h v}+O_{v h}+i O_{v v}}{2} \\
O_{12} & =\frac{O_{h h}-i O_{h v}+i O_{v h}+O_{v v}}{2} \\
O_{21} & =\frac{O_{h h}+i O_{h v}-i O_{v h}+O_{v v}}{2} \\
O_{22} & =\frac{i O_{h h}+O_{h v}+O_{v h}-i O_{v v}}{2} .
\end{aligned}
$$

Using (12)

$$
\begin{aligned}
& 2 O_{12}=O_{h h}-i O_{h v}+i O_{v h}+O_{v v}=\left(S_{h h}+S_{v v}\right) e^{-i 2 \Omega} \\
& 2 O_{21}=O_{h h}+i O_{h v}-i O_{v h}+O_{v v}=\left(S_{h h}+S_{v v}\right) e^{i 2 \Omega} .
\end{aligned}
$$

The FR angle is therefore given by

$$
\Omega=\frac{1}{4} \arg \left(O_{21} O_{12}^{*}\right)
$$

within the interval $-(\pi / 4) \leq \Omega \leq(\pi / 4)$.

In order to reduce the large dispersion of the estimate, it is usual to apply spatial averaging [36]. Since

$$
\begin{aligned}
4\left\langle O_{12} O_{21}^{*}\right\rangle= & \left(C_{11}+C_{14}+C_{41}+C_{44}+C_{23}+C_{32}\right) \\
& -\left(C_{22}+C_{33}\right)+i\left(C_{31}+C_{13}+C_{43}+C_{34}\right) \\
& -i\left(C_{21}+C_{12}+C_{42}+C_{24}\right)
\end{aligned}
$$

it follows from (14) and (15) that

$\left\langle O_{12} O_{21}^{*}\right\rangle=(\cos 4 \Omega+i \sin 4 \Omega) \frac{\rho_{H H}+2 \Re\left\{\rho_{H V}\right\}+\rho_{V V}}{4}$

2) Freeman's First Estimator: Freeman's “first" estimator uses the difference between the two cross-polarized channels of the scattering matrix in the linear basis [38]. From (12), it follows that

$$
\begin{aligned}
O_{h v}-O_{v h} & =2\left(S_{h h}+S_{v v}\right) \sin \Omega \cos \Omega=\left(S_{h h}+S_{v v}\right) \sin 2 \Omega \\
O_{h h}+O_{v v} & =\left(S_{h h}+S_{v v}\right)\left(\cos ^{2} \Omega-\sin ^{2} \Omega\right) \\
& =\left(S_{h h}+S_{v v}\right) \cos 2 \Omega .
\end{aligned}
$$

Therefore

$$
\Omega=\frac{1}{2} \tan ^{-1} \frac{O_{h v}-O_{v h}}{O_{h h}+O_{v v}} .
$$

Equation (23) allows the FR to be estimated in the interval between $-(\pi / 4)$ and $(\pi / 4)$.

3) Freeman's Second Estimator: To reduce the impact of additive noise on the first Freeman estimator, Freeman proposed an alternative estimator based on the covariance matrix [38]

$\Omega= \pm \frac{1}{2} \tan ^{-1} \sqrt{\frac{\left\langle O_{h v} O_{h v}^{*}\right\rangle+\left\langle O_{v h} O_{v h}^{*}\right\rangle+2 \Re\left\langle O_{h v} O_{v h}^{*}\right\rangle}{\left\langle O_{h h} O_{h h}^{*}\right\rangle+\left\langle O_{v v} O_{v v}^{*}\right\rangle+2 \Re\left\langle O_{h h} O_{v v}^{*}\right\rangle}}$.

The numerator and the denominator in the square root of (24) are given by the mean powers of those in (23). Using (14) and (15), the numerator in the square root becomes

$C_{33}-C_{23}-C_{32}+C_{22}=\left(\rho_{H H}+2 \Re\left\{\rho_{H V}\right\}+\rho_{V V}\right) \sin ^{2} 2 \Omega$ 
and the denominator is

$$
C_{11}+C_{44}+C_{14}+C_{41}=\left(\rho_{H H}+2 \Re\left\{\rho_{H V}\right\}+\rho_{V V}\right) \cos ^{2} 2 \Omega
$$

thus allowing the FR angle to be estimated between 0 and $\pi / 4$.

Although the estimator reduces the impact of additive noise, it yields a biased estimate of $\Omega$. Indeed, the $O_{h v}-O_{v h}$ term of (23) can be written as $O_{h v}-O_{v h}=z \sin 2 \Omega+n_{z}$, where $z=S_{H H}+S_{V V}$ from (12), and $n_{z}$ is a Gaussian random noise component, leading to the numerator of (24) to be

$$
\begin{aligned}
& \left\langle O_{h v} O_{h v}^{*}\right\rangle+\left\langle O_{v h} O_{v h}^{*}\right\rangle-2 \Re\left\langle O_{h v} O_{v h}^{*}\right\rangle \\
& \quad=\left\langle z z^{*}\right\rangle \sin ^{2} 2 \Omega+2 \Re\left\{z n_{z}^{*}\right\} \sin 2 \Omega+\left\langle n_{z} n_{z}^{*}\right\rangle .
\end{aligned}
$$

Similarly, the denominator is

$$
\begin{aligned}
& \left\langle O_{h h} O_{h h}^{*}\right\rangle+\left\langle O_{v v} O_{v v}^{*}\right\rangle+2 \Re\left\langle O_{h h} O_{v v}^{*}\right\rangle \\
& \quad=\left\langle z z^{*}\right\rangle \cos ^{2} 2 \Omega+2 \Re\left\{z n_{o}^{*}\right\} \cos 2 \Omega+\left\langle n_{o} n_{o}^{*}\right\rangle
\end{aligned}
$$

where $n_{O}$ is a noise contribution that shares the same statistics with $n_{z}$. Although $\Re\left\{z n_{z}^{*}\right\}$ and $\Re\left\{z n_{o}^{*}\right\}$ converge to zero with increasing number of looks, $\left\langle n_{z} n_{z}^{*}\right\rangle$ and $\left\langle n_{o} n_{o}^{*}\right\rangle$ converge to the same noise variance at a common positive level. As a result, when $-(\pi / 8)<\Omega<(\pi / 8)$, (24) tends to overestimate FR. Note that this condition would be violated often in the case of spaceborne P-band SAR configurations [40]. In such cases, FR will be underestimated.

4) Chen and Quegan Estimator: The Chen and Quegan estimator uses a $4 \times 4$ covariance matrix (13) that allows the derivation of six independent FR estimators based on the imaginary component of the covariance matrix elements [39]. The best performance is achieved by the third one of them

$$
\Omega=\frac{1}{2} \arg \left(\Im\left\{C_{14}\right\}+\frac{i}{2} \Im\left\{C_{12}+C_{24}-C_{13}-C_{34}\right\}\right) .
$$

This is valid for $\Im\left\{\left\langle S_{h h} S_{v v}^{*}\right\rangle\right\} \geq 0$, and for $\Im\left\{\left\langle S_{h h} S_{v v}^{*}\right\rangle\right\}<0$, $\Omega+(\pi / 2)$ is estimated, where $\Im$ denotes the imaginary part of a complex number. The real and imaginary parts of (29) can be written with respect to the original covariance matrix elements as

$$
\begin{aligned}
\Im\left\{C_{14}\right\} & =\Im\left\{\rho_{H V}\right\} \cos 2 \Omega \\
\Im\left\{C_{12}-C_{13}\right\} & =\Im\left\{C_{24}-C_{34}\right\}=\Im\left\{\rho_{H V}\right\} \sin 2 \Omega
\end{aligned}
$$

using (14) and (15), respectively. Accordingly, the Chen and Quegan estimator estimates the FR between $-(\pi / 2)$ and $\pi / 2$ [39].

\section{Performance}

The performances of the FR estimators depend on the SNR level in the data and on the number of independent samples used by the estimator (also known as the number of looks). While the total power of fully polarimetric observation does not change under FR, the power of the individual elements of scattering or covariance matrices used in (12), (14), and (15) does. As the different estimators introduced in the previous section rely on different elements of the scattering and covariance matrices, the performance of the FR estimator will not be identical.

The Bickel and Bates and Freeman's estimators are based on the $\left(\rho_{H H}+2 \Re\left\{\rho_{H V}\right\}+\rho_{V V}\right) / 4$ term [see (21), (25), and (26)], whereas the Chen and Quegan estimator is based on the $\Im\left\{\rho_{H V}\right\}$ term [see (30)]. However, the power of the $\left(\rho_{H H}+\right.$ $\left.2 \Re\left\{\rho_{H V}\right\}+\rho_{V V}\right) / 4$ term, which uses a large portion of total power (TP $=\left|S_{h h}\right|^{2}+2\left|S_{x x}\right|^{2}+\left|S_{v v}\right|^{2}$ for single-look data, or $\mathrm{TP}=\rho_{H H}+2 \rho_{X X}+\rho_{V V}=\operatorname{tr}(\mathbf{C})$ for multilook data), is larger than the power of the $\Im\left\{\rho_{H V}\right\}$ term. The HH-VV coherence is lower than 1 , and the imaginary part comprises only a fraction of the available power. For example, the power of $\left(\rho_{H H}+2 \Re\left\{\rho_{H V}\right\}+\rho_{V V}\right) / 4$ term over tropical forest (ALOS scene ID 065127070) is about $-8.3 \mathrm{~dB}$, whereas that of $\Im\left\{\rho_{H V}\right\}$ term is only $-16.8 \mathrm{~dB}$. This trend is the same for a Bragg scattering dominated scene (ALOS scene ID 069761410) where they are -12.2 and $-26.6 \mathrm{~dB}$, respectively. On the other hand, the noise contributions are similar as they do not significantly vary in the different polarization channels. Without a careful estimation of the noise power in each term, we can therefore conclude that the Bickel and Bates and Freeman's estimators make use of a higher SNR than the Chen and Quegan estimator.

In the single-look case, the performance of Freeman's first estimator is the same as that of the Bickel and Bates estimator; in the multilook case, Freeman's first estimator performs slightly worse [36], [41]. In the following, only the performance of the Bickel and Bates estimator will be analyzed because of its superior performance.

The variance of the FR estimates obtained by means of (19) depends on the polarimetric coherence between $O_{12}$ and $O_{21}$ that depends (only) on the SNR level [42], [43]

$$
\sigma_{\Omega}^{2}=\frac{1}{16}\left(\frac{\pi^{2}}{3}-\pi \sin ^{-1} \gamma_{\mathrm{SNR}}+\left(\sin ^{-1} \gamma_{\mathrm{SNR}}\right)^{2}-\frac{\mathrm{Li}_{2}\left(\gamma_{\mathrm{SNR}}^{2}\right)}{2}\right)
$$

where the noise-induced decorrelation $\gamma_{\mathrm{SNR}}$ is given by

$$
\gamma_{\mathrm{SNR}}=\frac{S N R}{(1+S N R)}
$$

and $\mathrm{Li}_{2}$ is the Euler dilogarithm. The coefficient of 1/16 follows directly from (19) where $\Omega$ is given by the $1 / 4$ of the cross-polar phase difference. As the $O_{12}$ and $O_{21}$ terms in (18) indicate signal channels with strong power, $\gamma_{\mathrm{SNR}}$ is close to one. For high $\gamma_{\mathrm{SNR}}$, the standard deviation of FR estimates $\sigma_{\Omega}$ reduces.

FR is estimated more accurately using spatial averaging (i.e., multilooking) [44]

$$
\sigma_{\Omega}^{2}=\frac{1}{16} \frac{1-\gamma_{\mathrm{SNR}}^{2}}{2 \gamma_{\mathrm{SNR}}^{2} L}
$$

where $L$ is the number of looks. Equation (33) assumes distributed scatterers, whereas (31) is valid only for point scatterers. This explains why, for $L=1$, (33) does not reproduce the same values of $\sigma_{\Omega}$ as given in (31). In Table I, the values of $\sigma_{\Omega}$ for different numbers of looks $(1,1000$, and 10000$)$ for $\gamma_{\mathrm{SNR}}$ of 0.99 are presented. In the single-look case, $\sigma_{\Omega}$ is derived from (31), and (33) is applied for a larger number of looks. For a large number of looks, the estimates of FR become very accurate. 
TABLE I

EXPECTED STANDARD DEVIATIONS OF FR AND TEC ESTIMATES FOR LOOKS

\begin{tabular}{llll}
\hline \hline \multicolumn{1}{c}{ Number of looks } & \multicolumn{1}{c}{1} & \multicolumn{1}{c}{1,000} & \multicolumn{1}{c}{10,000} \\
\hline$\sigma_{\Omega}$ (rad.) & 0.0659 & 0.000797 & 0.000252 \\
$\sigma_{\Omega}$ (deg.) & 3.77 & 0.0456 & 0.0144 \\
$\sigma_{\text {TEC }}$ (TECU) & 11.2 & 0.136 & 0.0429 \\
\hline \hline
\end{tabular}

SNR decorrelation $\gamma_{S N R}$ of 0.99 is assumed. For the conversion to the TEC, the P-band center frequency (435 MHz) and $\vec{B} \cdot \hat{\kappa}=40,000 \mathrm{nT}$ are applied.

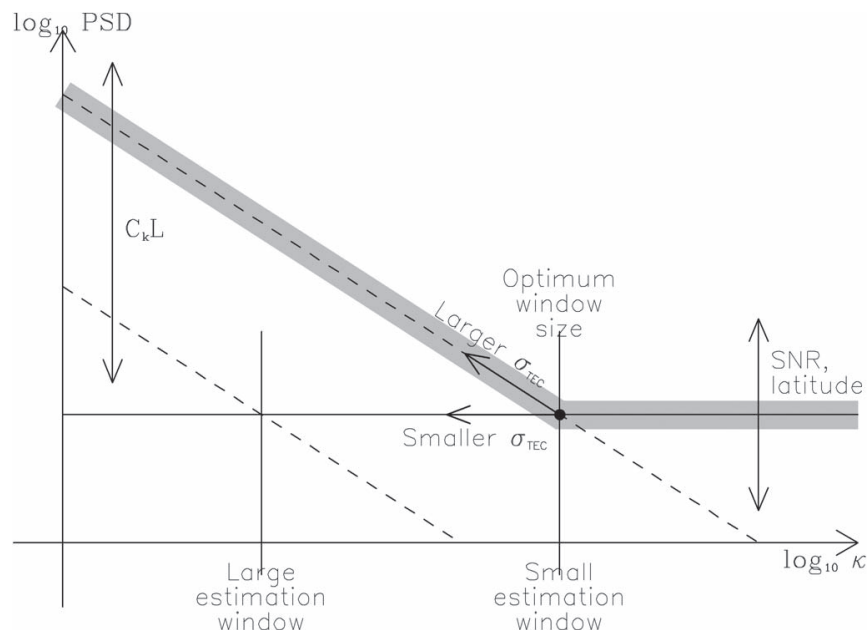

Fig. 4. Graphical representation of the tradeoff between the strength of irregularities and the noise-induced variance of the TEC estimation for the selection of the optimal FR estimation window size.

\section{Resolution}

At a given frequency $f$, the knowledge of the parallel geomagnetic field component $\vec{B} \cdot \hat{\kappa}$ allows converting the estimated FR to TEC using (7). As indicated by (33) and shown in Table I, at P-band, TEC estimation can be quite accurate, particularly at polar regions (with $\vec{B} \cdot \hat{\kappa} \geq 40000 \mathrm{nT}$ ). However, in order to reach this accuracy, a large number of looks are required, which hinders estimation of the (fine) spatial structure of the ionospheric irregularities. This tradeoff between estimation accuracy and the size of the estimation window is discussed in the following.

In Fig. 4, the (logarithm of the) power spectral distribution (PSD) of TEC is plotted against the (logarithm of the) spatial wavenumber $\kappa$ (where $\kappa=1 /$ Length). As discussed in Section III-C, the spatial scale of the ionospheric irregularities is characterized by a power law, leading to a straight line (dashed in Fig. 4) on the log-log plot. While the slope of the PSD line does not greatly vary with changing $C_{k} L$ (which defines the strength of the irregularities), the $y$-intersection of the line does. For qualitative analysis of the optimum resolution, the slope is approximated to be constant. Accordingly, the PSD line is shifted in the vertical direction depending on the strength of the scintillation.

In contrast to the PSD of TEC, the PSD of a white noise process plotted in Fig. 4 is a horizontal solid line, which is shifted toward higher $y$-values with increasing noise power. With increasing spatial wavenumber, the PSD of the estimated TEC map will follow the line associated with the actual $C_{k} L$ level (physics-dominated regime) until this crosses the hori- zontal line that corresponds to the noise level associated to the variance of the TEC estimates (induced by the variance of the corresponding FR estimates). As the spatial wavenumber further increases, the PSD of the estimated TEC levels off and follows the horizontal white noise line (noise-dominated regime). The spatial wavenumber at the intersection point will be called as the critical irregularity wavenumber in the following, and its length will be referred as the critical irregularity wavelength. The optimum estimation window size is defined by the critical irregularity wavelength: Larger estimation windows will blur the ionospheric structures, whereas smaller window sizes provide a suboptimal noise suppression.

With decreasing SNR level, the noise equivalent $C_{k} L$ level increases, pushing the intersection point into the direction indicated by "Larger $\sigma_{\text {TEC }}$ " in Fig. 4 . The new intersection point is at a larger critical irregularity wavelength (i.e., smaller $\kappa$ ), indicating a larger optimal estimation window. This is required in order to reduce the increased variance induced by the lower SNR level. However, at the same time, the larger window increases the blurring of the fine ionospheric structure leading to an overall worsening TEC estimation performance. In contrast, with decreasing $C_{k} L$ level, the PSD of the TEC moves downward, shifting the intersection point into the direction indicated by "Smaller $\sigma_{\mathrm{TEC}}$ " in Fig. 4. The associated larger optimal estimation window allows a better suppression of the noise-induced variance. In this case, the overall TEC estimation performance improves.

While in the first scenario (i.e., decreased SNR), the FR (and, therefore, TEC) estimation performance degrades, in the second scenario (i.e., decreased $C_{k} L$ ), better TEC estimation occurs. However, in both cases, the dimension of the estimation window increases. For the decreased SNR level scenario, the larger estimation window reduces the variance of the FR estimates and, at the same time, averages out the ionospheric irregularities. In the decreasing $C_{k} L$ level scenario, the increase in the estimation window size does not cause any additional degradation of the TEC estimation as the ionospheric irregularities are less severe.

\section{Simulation ANd CorRection of SCINTILlation}

\section{A. Ionospheric Phases From FR}

Using (3), the ionospheric phase screen can be obtained from the TEC map, which itself can be derived from the estimated FR using (7). Combining (3) and (7), we can write

$$
\phi_{\text {iono }}=\frac{4 \pi m_{e} f}{e \vec{B} \cdot \hat{\kappa}} \Omega .
$$

Assuming that $\vec{B} \cdot \hat{\kappa}=40000 \mathrm{nT}$, the proportionality factor is equal to 2269 at L-band $(1.27 \mathrm{GHz})$ and 777 at P-band (435 MHz) [45] and decreases with increasing latitude (see Fig. 1 in Section II-B). The standard deviation of the ionospheric phase screen $\sigma_{\phi}$ at a given location is defined by that of FR $\sigma_{\Omega}$. Accordingly, $\sigma_{\phi}$ reduces as the number of looks increases (33) and/or latitude increases. This is shown in Table II where $\sigma_{\phi}$ values are given for both P- and L-bands, assuming that $\gamma_{\mathrm{SNR}}=0.99$ and $L=1000$. One can see that, at 
TABLE II

STANDARd DEVIATION OF THE IONOSPHERIC PHASE SCREEN For DIFFERENT VALUES OF $\vec{B} \cdot \hat{\kappa}$ FOR A $\gamma_{\mathrm{SNR}}$ OF 0.99 AND 1000 LOOKS

\begin{tabular}{cccc}
\hline \hline $\begin{array}{c}\text { Parallel } \\
\text { geomagnetic }\end{array}$ & $\begin{array}{c}\text { Approximated } \\
\text { geographic }\end{array}$ & \multicolumn{2}{c}{$\begin{array}{c}\text { Deviation of ionospheric } \\
\text { phase } \sigma_{\phi}\end{array}$} \\
field $\vec{B} \cdot \hat{\kappa}$ & latitude & P-band & L-band \\
\hline $40,000 \mathrm{nT}$ & $70^{\circ} \mathrm{N}, 60^{\circ} \mathrm{S}$ & $35.5^{\circ}$ & $105^{\circ}$ \\
$30,000 \mathrm{nT}$ & $50^{\circ} \mathrm{N}, 40^{\circ} \mathrm{S}$ & $47.3^{\circ}$ & $140^{\circ}$ \\
$20,000 \mathrm{nT}$ & $30^{\circ} \mathrm{N}, 20^{\circ} \mathrm{S}$ & $70.9^{\circ}$ & $210^{\circ}$ \\
$10,000 \mathrm{nT}$ & $15^{\circ} \mathrm{N}, 10^{\circ} \mathrm{S}$ & $142^{\circ}$ & $421^{\circ}$ \\
$5,000 \mathrm{nT}$ & $5^{\circ} \mathrm{N}, 5^{\circ} \mathrm{S}$ & $284^{\circ}$ & $841^{\circ}$ \\
\hline \hline
\end{tabular}

P-band at higher latitudes (i.e., $\vec{B} \cdot \hat{\kappa} \geq 30000 \mathrm{nT}$ ), $\sigma_{\phi}$ levels smaller than $\pi / 4$ are feasible.

\section{B. Correction Method}

The azimuth phase history of a point-like scatterer can be approximated by a quadratic function (see Fig. 2)

$$
\phi(t)=\frac{4 \pi}{\lambda} \sqrt{R_{0}^{2}+(v t)^{2}} \approx \frac{4 \pi R_{0}}{\lambda}+\frac{2 \pi}{\lambda R_{0}} v^{2} t^{2}
$$

where $R_{0}$ is the shortest range between the sensor and the scatterer (i.e., the zero-Doppler distance). The first time derivative of the phase [identical to (8)]

$$
f_{a}(t)=\frac{2 v^{2} t}{\lambda R_{0}}=\frac{2 v^{2} f}{R_{0} c_{0}} t=D_{f} t
$$

is known as the time-Doppler relation [46, p. 168]. Hence, (36) can be written as

$$
\phi\left(f_{a}, R_{0}\right)=\frac{4 \pi}{\lambda} R_{0} \sqrt{1+\left(\frac{f_{a} \lambda}{2 v}\right)^{2}} .
$$

By substituting $R_{0}$ by some arbitrary distance $R$ in (37), it is possible to vary the altitude of the focusing plane, as only the contributions with a phase history matching (37) are properly focused in the azimuth direction [47]. Accordingly, starting from the SLC image focused with respect to the distance $R=$ $R_{0}$, it is possible to obtain an image focused at the ionospheric altitude $S L C_{\text {iono }}$ by: 1) Fourier transforming the SLC image into the frequency domain; 2) decompressing in the azimuth direction by multiplying by $\left.e^{-i \phi\left(f_{a}, R_{0}\right)} ; 3\right)$ partially focusing back to the ionospheric altitude by multiplying by $e^{i \phi\left(f_{a}, R_{\text {iono }}\right)}$, where $R=R_{\text {iono }}$ is the range to the ionospheric layer (after range focusing and range migration compensation); and, finally, 4) using an inverse Fourier transform to get back to the image domain

$$
\mathcal{F}\left(S L C_{\text {iono }}\right)=\mathcal{F}(S L C) \cdot e^{-i \phi\left(f_{a} R_{0}\right)} \cdot e^{i \phi\left(f_{a}, R_{\text {iono }}\right)} .
$$

An example is shown in Fig. 5. The top image is an ALOS PALSAR SLC Pauli basis RGB composite (blue for $\mathrm{HH}+\mathrm{VV}$, red for $\mathrm{HH}-\mathrm{VV}$, and green for $\mathrm{HV}=\mathrm{VH}$ ) image focused at ground level, whereas the bottom image is the same image but focused at the height of the ionospheric layer assumed to be approximately at, i.e., 200-km altitude. The lower image appears blurred in azimuth because of the mismatch between the
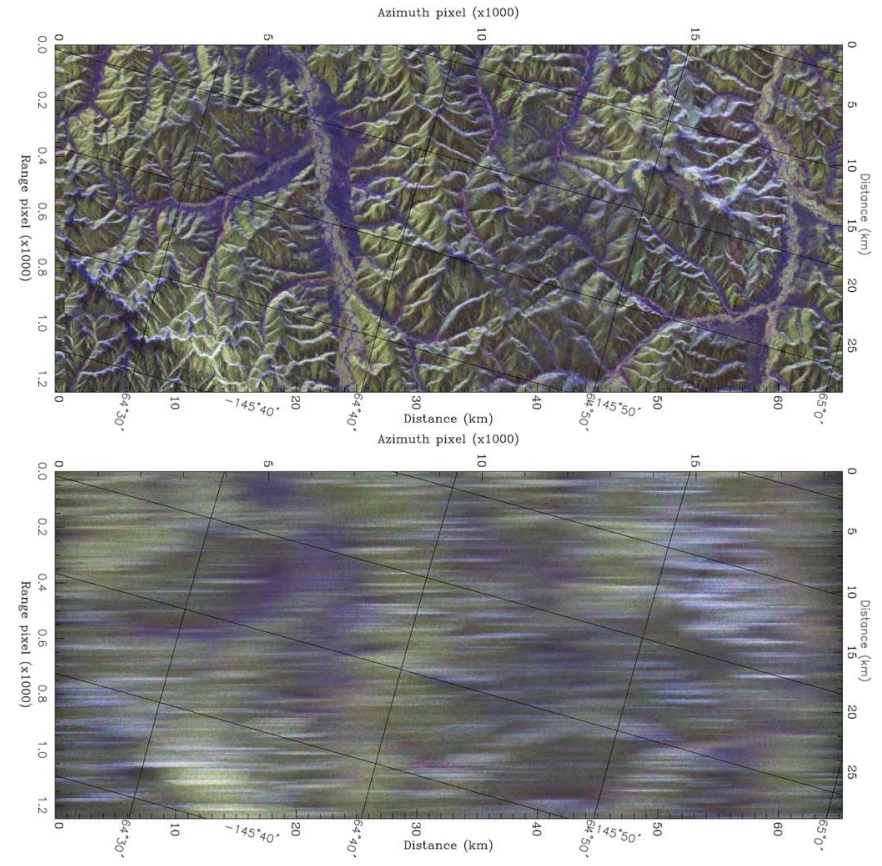

Fig. 5. Pauli images focused at ground level (top) and at the height of the ionospheric layer, assumed to be at $200-\mathrm{km}$ altitude.

time-Doppler relations of ground scatterers and the matched filter at the ionospheric level.

Once the SLC is focused at the ionospheric altitude, the ionospheric phase screen corresponds to the TEC map at that altitude and can be compensated by multiplying $e^{-i \phi_{\text {iono }}}$, where $\phi_{\text {iono }}$ is obtained from (3)

$$
S L C_{\text {iono }}^{\prime}=S L C_{\text {iono }} \cdot e^{-i \phi_{\text {iono }}} .
$$

Finally, the corrected SLC image focused at ground level is obtained by reversing the operations in (38)

$$
\mathcal{F}\left(S L C^{\prime}\right)=\mathcal{F}\left(S L C_{\text {iono }}^{\prime}\right) \cdot e^{-i \phi\left(f_{a}, R_{\text {iono }}\right)} \cdot e^{i \phi\left(f_{a}, R_{0}\right)} .
$$

This corrects for 1) the ionospheric phase advance and also compensates 2) the ionospheric azimuth shift. The phases from the TEC gradient inducing (azimuth) shifts are compensated by (39). Its performance is determined by the accuracy of the estimated phase screen $\phi_{\text {iono }}$. The correction scheme is summarized in Fig. 6.

Note that the FR is estimated from the defocused SLC. This not only ensures consistency between the SLC and FR estimates but also enhances the spatial resolution of the FR estimates.

\section{DATA ANALYsis}

In this section, we validate the proposed scintillation correction methodology by applying it to simulated spaceborne P-band data (as real spaceborne P-band data are unavailable) and real L-band ALOS PALSAR data. Using well-characterized simulated data means that the impact of scintillation and the performance of the correction at P-band can be accurately accessed in terms of the cross-correlation coefficient between the original and the distorted or the corrected data. 


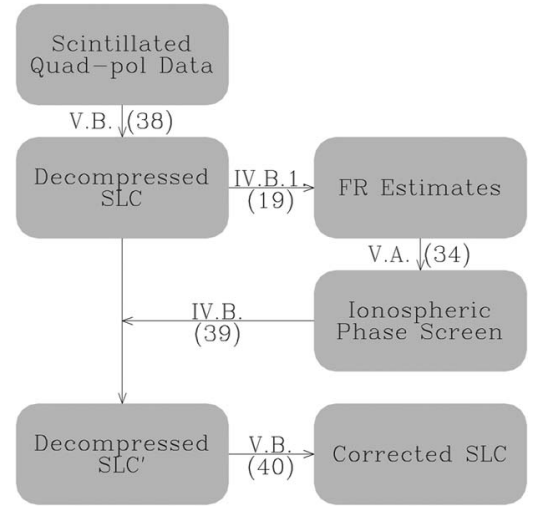

Fig. 6. Flowchart of the scintillation correction algorithm. The relations of processing steps (arrows) to section numbers and to equation numbers are indicated.

TABLE III

SYSTEM PARAMETERS OF E-SAR, BIOMASS, AND ALOS

\begin{tabular}{|c|c|c|c|}
\hline & E-SAR & BIOMASS & $\begin{array}{c}\text { ALOS PALSAR } \\
\text { (Fully polarimetric) }\end{array}$ \\
\hline Frequency $(\mathrm{MHz})$ & 350 & 435 & 1270 \\
\hline $\begin{array}{l}\text { Range bandwidth } \\
(\mathrm{MHz})\end{array}$ & 94 & 6 & 14 \\
\hline NESZ (dB) & -35 & -28 & -28 \\
\hline $\begin{array}{l}\text { Slant range } \times \\
\text { azimuth res. }(\mathrm{m} \times \mathrm{m})\end{array}$ & $1.6 \times 1.74$ & $25 \times 12.5$ & $10.7 \times 4.4$ \\
\hline $\begin{array}{l}\text { Slant range } \times \\
\text { azimuth pixel } \\
\text { spacing }(\mathrm{m} \times \mathrm{m})\end{array}$ & $1.5 \times 0.45$ & $21 \times 4.3$ & $9.4 \times 3.7$ \\
\hline
\end{tabular}

Correction at L-band is more challenging than at P-band as the proportionality factor in (34) is larger, requiring much more accurate FR estimation. Because of the absence of reference ALOS PALSAR scene (i.e., scintillation-free acquisition without temporal/geometric decorrelations), the correction performance is measured in terms of the interferometric coherence.

\section{A. Simulated BIOMASS P-Band Data}

1) Spaceborne Simulation: In order to assess the performance of the proposed methodology for a realistic P-band spaceborne scenario, the parameters of ESA's BIOMASS mission (see Table III) were used to degrade P-band data acquired by DLR's E-SAR airborne sensor over the Remningstorp site in Sweden on May 2, 2007 [47]. This involved three steps: 1) The spatial resolutions in slant range and azimuth were reduced by low-pass filtering the complex SAR data in the spectral domain, and the peak and integrated sidelobe ratios (PSLR and ISLR) were adjusted by removing the Hamming window usually used for optimum sidelobe suppression and instead applying a suitable cosine weighting; 2) the decrease in the noise equivalent sigma zero from $-35 \mathrm{~dB}$ in the airborne case to $-28 \mathrm{~dB}$ for BIOMASS [14] was simulated by adding zero-mean circular complex Gaussian noise; and 3) the range and the azimuth ambiguity levels were adjusted by adding attenuated, defocused, and spatially shifted image data from other (for range) and the same (for azimuth) polarizations to each channel (for more details, see [47]). For more convenient data handling, the pixel spacing is retained in the simulation.
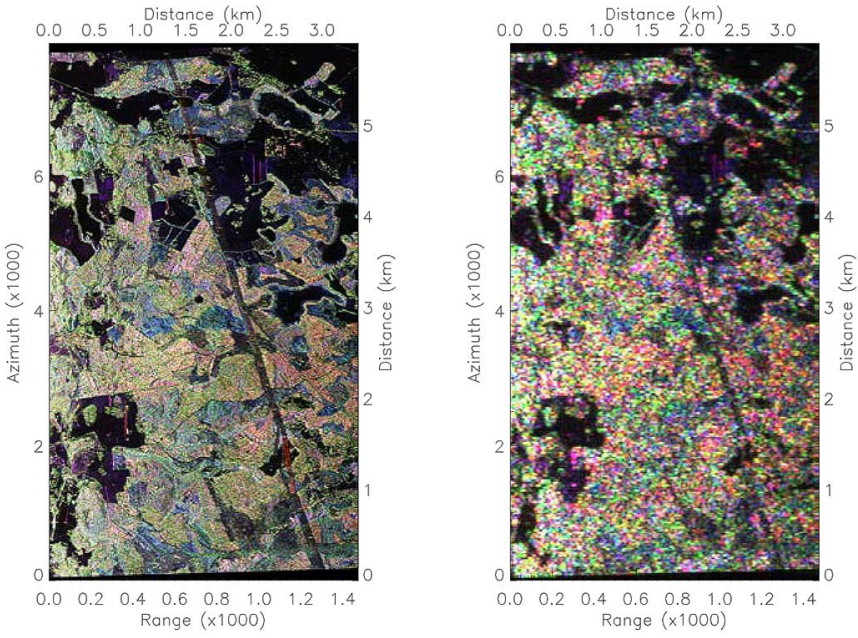

Fig. 7. P-band polarimetric RGB (HH-HV-VV) images of the Remningstorp site. (Left) Original E-SAR image (5-m multilook resolution). (Right) Simulated spaceborne (BIOMASS) image (50-m multilook resolution).

Fig. 7 compares the original E-SAR polarimetric RGB image on the left with a simulated spaceborne image on the right.

2) Simulation of Scintillation Phases: The ionospheric phase screens were generated using power law spectra derived from the wideband model (WBMOD) from NorthWest Research Associates [48], and the orbit parameters of the BIOMASS mission, i.e., $98^{\circ}$ inclination angle, $30^{\circ}$ off-nadir angle, and a sun-synchronous dusk-dawn orbit with local time of ascending node at 06:00. The calculations are performed for the summer solstice in 2000 (solar maximum) at a boreal location in North America $\left(65^{\circ} \mathrm{N}, 110^{\circ} \mathrm{W}\right)$. Scintillation associated with the auroral zone extends to its lowest latitude at this longitude because the Earth's North magnetic pole is offset from the geographic pole and lies on this meridian $\left(81^{\circ} \mathrm{N}, 110^{\circ} \mathrm{W}\right.$ in 2000) [19].

Six ionospheric phase screens were generated for different scintillation scenarios, corresponding to the 50th and 90th percentiles of $C_{k} L$ for each of the three planetary indices, i.e., $\mathrm{Kp}=1,3$, and 7. Since only scintillation effects are of concern here, the mean background ionosphere was set to zero. The standard deviations of the phase screens were $0.44,0.70$, and $1.15 \mathrm{rad}$ for $\mathrm{Kp}=1,3$, and 7 with 50th percentiles of $C_{k} L$ and 1.37, 2.20, and 3.64 rad with 90th percentiles of $C_{k} L$.

3) Simulation of Image Distortion Due to Scintillation: To simulate image distortions due to scintillation, the scintillation correction approach in Section V-B was reversed. The ionospheric phase screens $\phi_{\text {iono }}$ (generated as described above) were applied as in (39) on the SAR data focused at the height of the ionospheric layer. The altitude of the ionospheric layer was taken to be $350 \mathrm{~km}$. Fig. 8 shows the SLCs before and after applying scintillation for $\mathrm{Kp}=1$ and $C_{k} L \mathrm{CI}=90 \%$ to the data. The effect of the azimuth defocusing (see Section III-C) is clearly visible. To measure the effect of the scintillations, the (complex) correlation coefficient $\rho$ between the undistorted and the distorted SLC images was calculated. Table IV summarizes the mean value of correlation coefficient amplitude $|\rho|$ across the image for the different scintillation scenarios: For stronger scintillations (larger $\mathrm{Kp}$ and/or $C_{k} L \mathrm{CI}$ values), the images become more distorted, leading to lower $|\rho|$ values. 

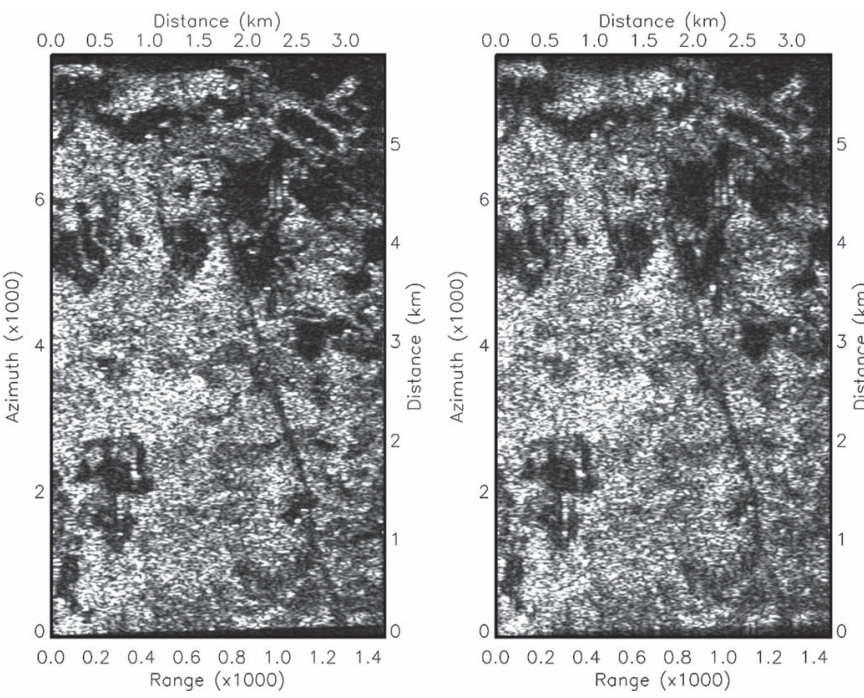

Fig. 8. (Left) Original and (right) distorted HH SLC images for $\mathrm{Kp}=1$ and $C_{k} L \mathrm{CI}=90 \%$.

TABLE IV

MEAn Correlation COEFFICIENT AMPlitudes BETWEEN ORIGINAL AND CORRUPTED SLCS

\begin{tabular}{lccc}
\hline \hline & $\mathrm{Kp} 1$ & $\mathrm{Kp} \mathrm{3}$ & $\mathrm{Kp} \mathrm{7}$ \\
\hline $\mathrm{C}_{\mathrm{k}} \mathrm{L} \mathrm{CI}=50 \%$ & 0.95 & 0.78 & 0.54 \\
$\mathrm{C}_{\mathrm{k}} \mathrm{L} \mathrm{CI}=90 \%$ & 0.41 & 0.17 & 0.33 \\
\hline \hline \multicolumn{4}{l}{ Estimated using 50 looks }
\end{tabular}

4) Simulation of FR: WBMOD provides the small-scale TEC variation and the associated ionospheric phase screens but not the corresponding FR arising from the background ionosphere. Simulation of the FR map was performed in two steps. First, the ionospheric phase screens were converted to FR maps using (34); the associated values were no more than a few degrees, even for the strong scintillation scenarios. Second, in order to obtain a realistic noise-affected FR map, a zero-mean normal distributed FR screen was added, with variance defined by the SNR (31). To simulate multilooked FR estimates, the obtained FR maps were spatially averaged. The correction was then based on the estimated FR maps, which were converted to phase screens using (34).

For each ionospheric screen, estimated FR maps were generated for two SNR levels, i.e., 18 and $13 \mathrm{~dB}$ (corresponding to $\gamma_{\mathrm{SNR}}$ of 0.984 and 0.952 , respectively); three numbers of looks $L$, i.e., 1600,3600 , and 6400 (corresponding to window sizes of $1 \mathrm{~km} \times 1 \mathrm{~km}, 1.5 \mathrm{~km} \times 1.5 \mathrm{~km}$, and $2 \mathrm{~km} \times 2 \mathrm{~km}$, respectively); and two values of $\vec{B} \cdot \hat{\kappa}$, i.e., 30000 and $40000 \mathrm{nT}$. For the assumed location of $\left(65^{\circ} \mathrm{N}, 110^{\circ} \mathrm{W}\right), \vec{B} \cdot \hat{\kappa}$ is about $44000 \mathrm{nT}$ at $350-\mathrm{km}$ altitude. Here, the same set of ionospheric phase screens has been used as for the $\vec{B} \cdot \hat{\kappa}=30000 \mathrm{nT}$ case, which corresponds to a mid-latitude location. However, scintillations are far rarer at such latitudes [4], and in this case, the scintillations imposed are unrealistically strong.

Fig. 9 shows an example of (top) an original phase screen for $\mathrm{Kp}=1, C_{k} L \mathrm{CI}=90 \%, \mathrm{SNR}=13 \mathrm{~dB}$, and $\vec{B} \cdot \hat{\kappa}=$ $40000 \mathrm{nT}$ and (bottom) the estimated phase screen using 1600 looks. This level of multilooking does not give very good estimates since the noise level is insufficiently reduced.
5) Correction Performance: The correction performance strongly depends on the quality of the phase screen constructed from the estimated FR map. This, in turn, depends on the SNR, the size of the estimation window, $\vec{B} \cdot \hat{\kappa}$, and ionospheric conditions, as summarized in the four plots in Fig. 10. The $x$-axes indicate the size of the estimation window used to estimate $\mathrm{FR}$, and the $y$-axes give the correlation coefficient amplitude between the undisturbed and corrected SLC images. The plots in the left and right columns are for SNR levels of $18 \mathrm{~dB}\left(\sigma_{0}=-10 \mathrm{~dB}\right)$ and $13 \mathrm{~dB}\left(\sigma_{0}=-15 \mathrm{~dB}\right)$, respectively. The plots in the top and bottom rows are for $\vec{B} \cdot \hat{\kappa}$ values of 40000 and $30000 \mathrm{nT}$, respectively. The six colors in each plot indicate different ionospheric conditions (violet, blue, and cyan for $\mathrm{Kp}=1,3$, and 7 with $C_{k} L \mathrm{CI}=50 \%$, and yellow, orange, and red for the same Kp's but $C_{k} L \mathrm{CI}=90 \%$ ). The arrows on the right $y$-axis mark the correlation coefficient amplitudes before ionospheric correction (as in Table IV). The horizontal thick lines show where the corrected correlation coefficient amplitude exceeds that of the uncorrected data; it is shown that the correction sometimes worsens the image quality.

The information in the plots can be summarized as follows.

- The correlation coefficient amplitude increases for larger $\vec{B} \cdot \hat{\kappa}$ because of the reduced variance in FR [see (34)].

- The correction performance worsens as the $\mathrm{Kp}$ and $C_{k} L$ CI levels increase and improves as SNR increases.

- The impact of the number of looks used to estimate FR is more complex. For low ionospheric activity, i.e., $C_{k} L \mathrm{CI}=50 \%$, larger estimation windows give better correction but not for strong ionospheric activity, i.e., $C_{k} L \mathrm{CI}=90 \%$ and $\mathrm{Kp}=7$. This can be explained using the critical irregularity wavelength concept introduced in Section IV-D. For calm ionospheric conditions, the critical irregularity wavelength is larger than all three estimation windows used in the simulations; thus, increasing the size of the window improves the correction. For higher $C_{k} L$ levels, the critical irregularity wavelength decreases and the optimum window size becomes smaller. As an example, for $C_{k} L \mathrm{CI}=90 \%$ and $\mathrm{Kp}=7$ (red curve), the critical irregularity wavenumber is around $1 /(1.5 \mathrm{~km})$ for $\sigma_{0}=-15 \mathrm{~dB}$ and $\vec{B} \cdot \hat{\kappa}=40000 \mathrm{nT}$ (top right in Fig. 10). With increasing SNR $\left(\sigma_{0}=-10 \mathrm{~dB}\right.$, top left in Fig. 10), the PSD level of the noise reduces and the critical irregularity wavelength is even less than $1 \mathrm{~km}$, as expected from Fig. 4.

6) Altitude Dependence: One other variable that affects the correction is the ionospheric altitude, which is used in the correction process (see Section V-B). In the following, we evaluate the reduction in performance caused by a mismatch between the true ionospheric altitude of $350 \mathrm{~km}$ used in the simulations and corrections with altitudes ranging from 100 to $600 \mathrm{~km}$ [achieved by adjusting $R_{\text {iono }}$ in (38) and (40)].

The correlation coefficient amplitude between the undisturbed and the corrected SLC is plotted in Fig. 11 as a function of the error in the knowledge of the ionospheric layer altitude for different ionospheric conditions. To separate the effect of the altitude mismatch from errors induced in FR estimation, the 

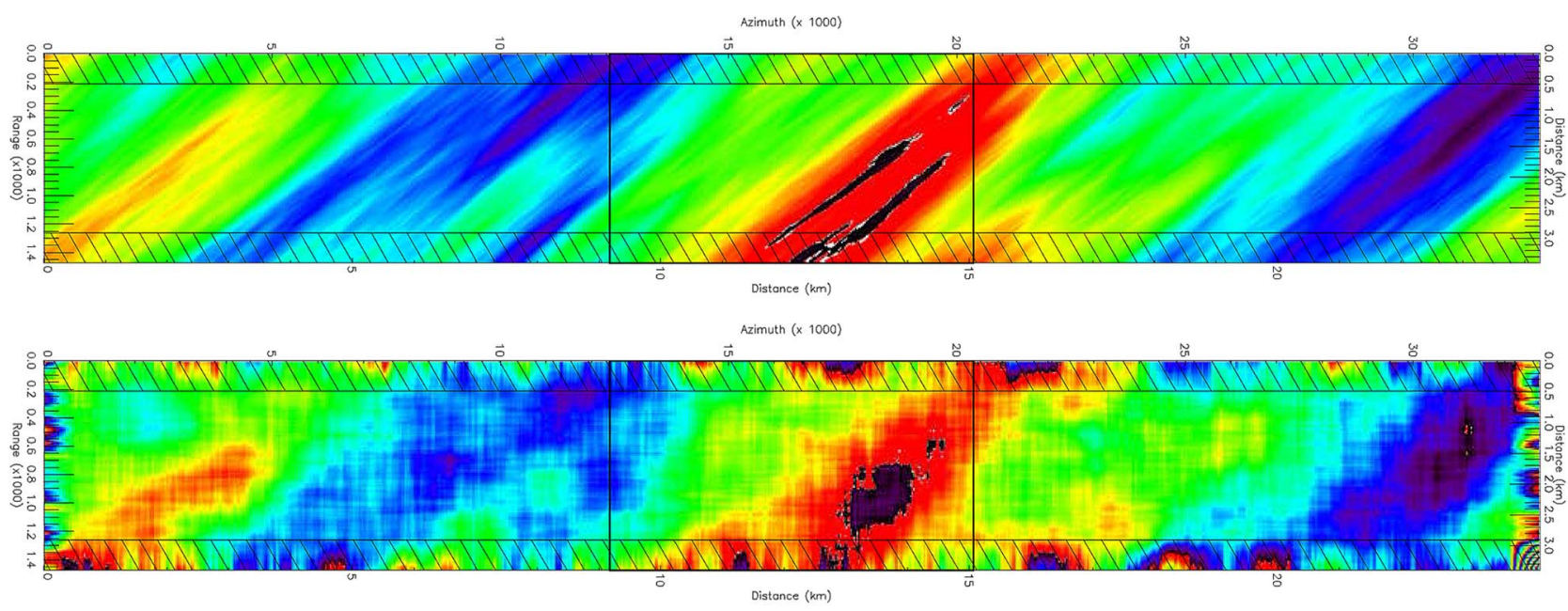

Fig. 9. (Top) Original ionospheric phase screen and after noise addition and spatial averaging of $1 \mathrm{~km} \times 1 \mathrm{~km}$ or (bottom) 1600 looks for $\mathrm{Kp} 1, C_{k} L \mathrm{CI}=90 \%$, SNR $=13 \mathrm{~dB}$, and $\vec{B} \cdot \hat{\kappa}=40000 \mathrm{nT}$. The shaded borders correspond to half the estimation window and indicate where spatial averaging was limited to a smaller number of samples.
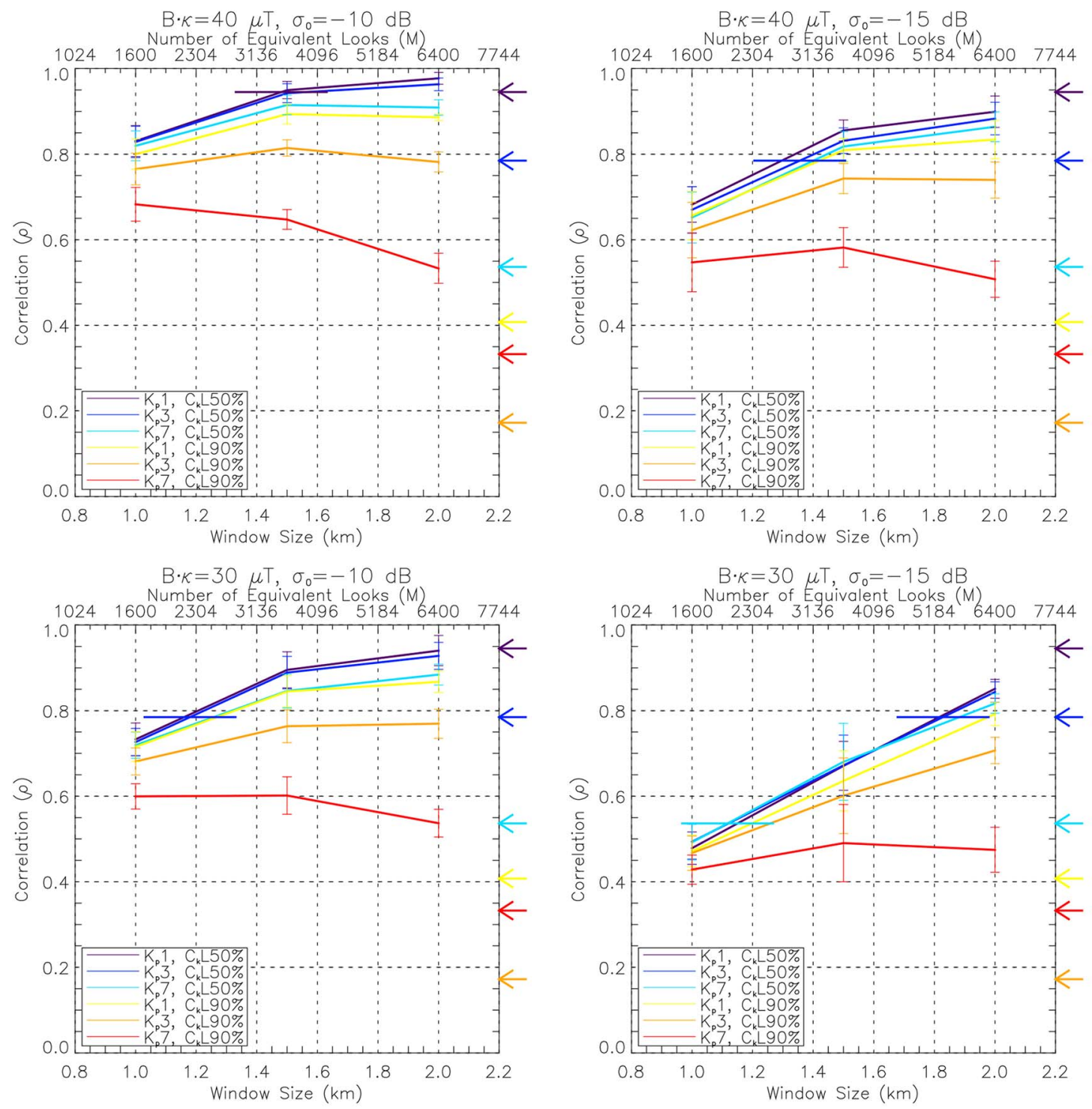

Fig. 10. Mean amplitude of the correlation between undisturbed and corrected SLCs plotted against the size of the estimation window for different ionospheric conditions, different SNR levels (left versus right column), and different values of $\vec{B} \cdot \hat{\kappa}$ (top versus bottom). The horizontal thick lines indicate where the corrected correlation coefficient amplitude exceeds that of the uncorrected data. 


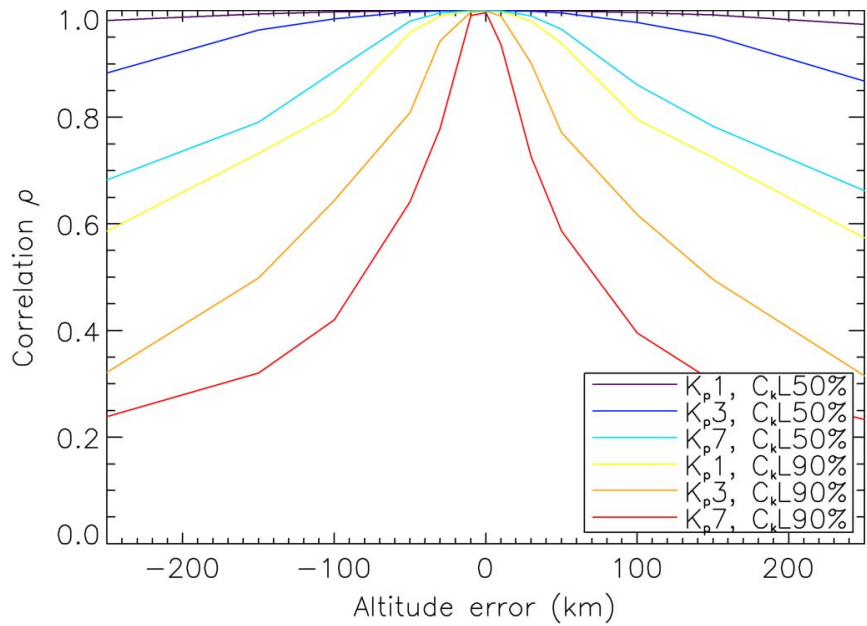

Fig. 11. Correction performance expressed by the mean correlation coefficient amplitude between undisturbed and corrected SLCs plotted against the error in ionospheric altitude estimation for different ionospheric conditions.

original ionospheric phase screens were applied for correction. Hence, $|\rho|=1$ for error-free altitude knowledge.

Fig. 11 indicates that the effect of altitude mismatch strongly depends on the ionospheric conditions. For calm conditions, the altitude error plays a minor role, but for stronger scintillations, the correction performance significantly reduces for even a few tens of kilometers of error. The trend in performance loss is almost symmetric for positive and negative errors.

7) Criteria for Applying Correction: It was already noted that the proposed correction does not always improve the image quality. Fig. 10 indicates that, for calm ionospheric conditions at lower latitudes, low SNR, and/or smaller number of looks, the mean correlation coefficient amplitude between the undisturbed and corrected SLCs can be less than the correlation between the undisturbed and disturbed SLCs. In this case, the variance of the estimated phase screen is larger than the perturbation induced by the scintillation itself, and correction reduces the image quality.

In order to provide an a priori evaluation of the expected performance, the correction algorithm is applied to undisturbed SLCs. The correlations between SLCs before and after correction are estimated for different values of $\vec{B} \cdot \hat{\kappa}$, SNR, and number of looks, and their amplitudes are plotted in Fig. 12 against the standard deviation of the ionospheric phases $\sigma_{\phi}$ estimated using (33) and (34). As expected, for smaller estimation windows, lower values of $\vec{B} \cdot \hat{\kappa}$, and lower SNR, the correlation decreases. Note that the correlation coefficient amplitudes in Fig. 12 generally agree well with those of the weakest scintillation condition $\left(\mathrm{Kp}=1, C_{k} L \mathrm{CI}=50 \%\right)$ in Fig. 10. Interestingly, the relation between $|\rho|$ and $\sigma_{\phi}$ is well fitted by a quadratic curve defined by

$$
|\rho|=1-\left(0.0545+0.0365 \cdot \sigma_{\phi}\right)^{2}
$$

where $\sigma_{\phi}$ is expressed in degrees. In the determination of (41), we accounted for that $|\rho|=1$ when $\sigma_{\phi}=0$, and (41) passes narrowly $(0,1)$. Equation (41) is useful for predicting the best

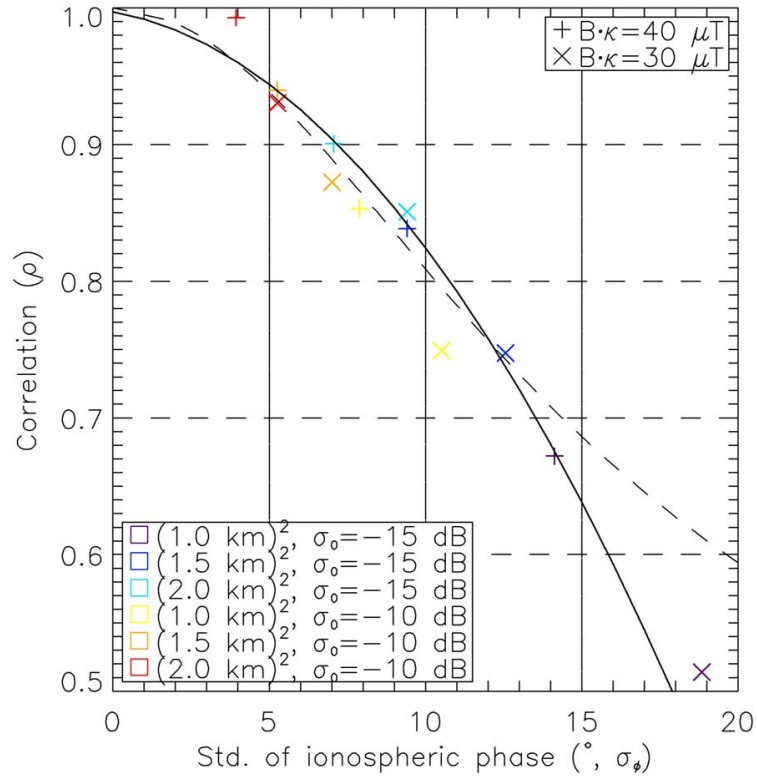

Fig. 12. Correction performance expressed by the mean correlation coefficient amplitude $|\rho|$ between the undisturbed and corrected SLC images plotted against the standard deviation of the ionospheric phases' estimates $\sigma_{\phi}$ for different geomagnetic latitudes, SNR levels, and number of looks $\left(1 \mathrm{~km}^{2}\right.$ corresponds to $1600,2.25 \mathrm{~km}^{2}$ to 3600 , and $4 \mathrm{~km}^{2}$ to 6400 looks). The solid curve is the best fit quadratic function, and the dashed curve is the conventional relation between the correlation (or coherence) and the standard deviation of the phase given by (33) for $L=10$.

performance of the correction methodology when the level of scintillation is unknown.

The dashed curve in Fig. 12 indicates the conventional relation between the coherence and the standard deviation of the phase given by $\sigma_{\phi}=\sqrt{\left(\left(1-|\gamma|^{2}\right) / 2|\gamma|^{2} L\right)}$ for $L=10$ number of looks. For thousand number of looks as in our cases, the dashed line would descent very fast as $\sigma_{\phi}$ increases, and this clearly fails to predict the relation between the correlation coefficient amplitude and the deviation of phase. We interpret that this discrepancy simply reflects the different physical and geometrical processes that each equation assumes. In the prediction of the performance of the proposed correction method, (41) fits better than the conventional relation between coherence and phase deviation.

\section{B. L-Band ALOS PALSAR}

1) Data Set: To assess the performance of the correction method on real SAR data, an ALOS L-band polarimetric interferometric pair acquired east of Fairbanks, Alaska, is used (see Fig. 5). A High Frequency Active Auroral Research Program (HAARP) ground station located within $200 \mathrm{~km}$ of the scene provides reference measurements; these indicate a strong ionospheric activity during the master acquisition on April 1, 2007, and a calm ionosphere for the slave acquisition on May 16, 2007 [49], [50].

The master and the slave images have a normal baseline of only $45 \mathrm{~m}$. The corresponding vertical wavenumber $k_{z}[51]$ is $0.008 \mathrm{rad} / \mathrm{m}$, equivalent to a height of ambiguity of about $790 \mathrm{~m}$ [51], so that the residual error due to digital elevation model 


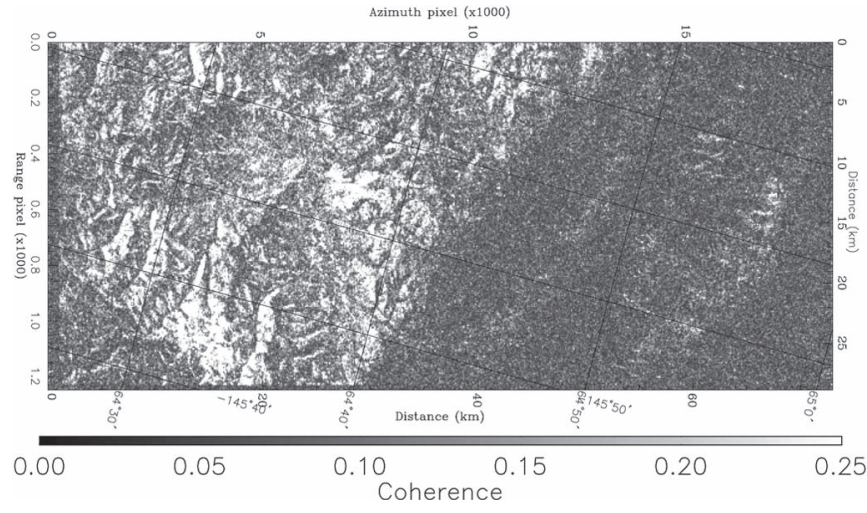

Fig. 13. Interferometric coherence map (HH-polarization) scaled linearly from (black) 0 to (white) 0.25 .

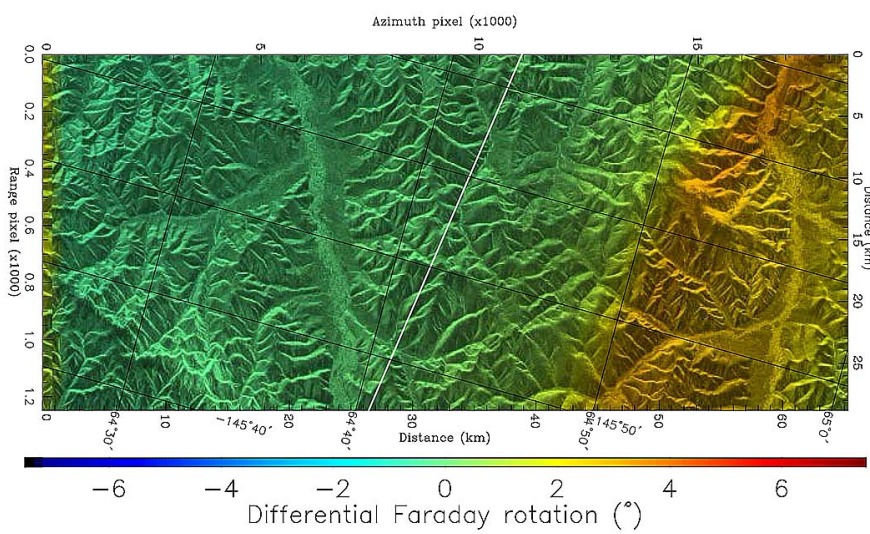

Fig. 14. Differential FR map superimposed on total power image. The white line indicates the projected magnetic field.

(DEM) inaccuracy when removing the topographic phase component from the interferogram can be neglected. The National Elevation Data DEM provided by the United States Geological Survey [52] was used for topographic phase correction of the interferogram. Fig. 13 shows the coherence map scaled from 0 (black) to 0.25 (white). Strong temporal decorrelation, probably due to snow melt and vegetation decorrelation, is evident over large parts of the scene. In the southern part (left side of the image), the coherence is about 0.25 , whereas in the northern part, the coherence is less than 0.05. A primary cause of the low coherence in the northern part is thought to be an ionospheric activity during the master acquisition, which induces azimuth shifts and decorrelation. Evidence for this is provided in Fig. 14 where the estimated differential FR $\left(\Omega_{\text {master }}-\Omega_{\text {slave }}\right)$ map is shown superimposed on the total power image. A stripe of high differential FR about $10 \mathrm{~km}$ wide crosses the northern part of the scene. The pattern of decorrelation in Fig. 13 is well aligned with the magnetic field line projected onto a horizontal plane at the expected ionospheric altitude of $200 \mathrm{~km}$ along the propagation vector $\hat{\kappa}$, which is indicated by the white line crossing Fig. 14. At this location, the Earth's magnetic field has magnitude of $51618 \mathrm{nT}$, and $\vec{B} \cdot \hat{\kappa}$ is $49070 \mathrm{nT}$ at an altitude of $200 \mathrm{~km}$. Under these conditions, $1^{\circ}$ of FR corresponds to 2.43 TECU [see (7)].

Fig. 15 shows the interferometric coherence after correction of the azimuth shift only for master, estimated using incoherent

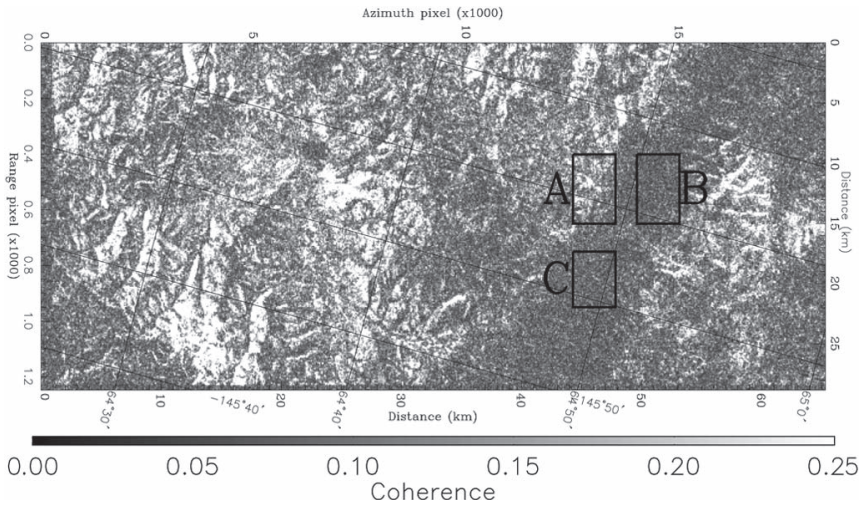

Fig. 15. Interferometric coherence map (HH-polarization) scaled from (black) 0 to (white) 0.25 after correction of variable azimuth shifts. Three rectangles $\mathrm{A}, \mathrm{B}$, and $\mathrm{C}$ are regions of interest for the coherence improvements using the ionospheric correction method.

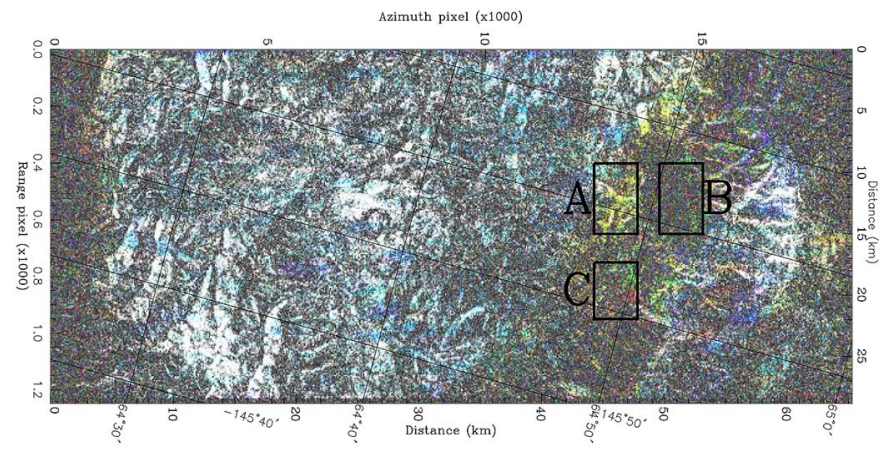

Fig. 16. RGB image of coherence values after scintillation correction using different estimation window sizes: red corresponds to $0.5 \mathrm{~km} \times 0.5 \mathrm{~km}$, green to $1 \mathrm{~km} \times 1 \mathrm{~km}$, and blue to $2 \mathrm{~km} \times 2 \mathrm{~km}$.

amplitude correlation. The low coherence makes coherent shift estimates inaccurate. However, also in the incoherent case, the distortion of the impulse response (as indicated by Fig. 3) makes the shift estimation in the areas of rapid TEC change suboptimal.

2) Number of Looks: When correcting the real SAR data, the parameter that controls the performance of the scintillation correction and can be chosen is the size of the estimation window. To demonstrate the impact of this choice, window sizes of $0.5 \times 0.5 \mathrm{~km}^{2}, 1 \times 1 \mathrm{~km}^{2}$, and $2 \times 2 \mathrm{~km}^{2}$, corresponding to 2800,11200 , and 44800 looks, respectively, were applied. The estimated FR maps were then used to correct the scintillations using the approach described in Section V-B; this simultaneously accounted for the azimuth shifts. The interferometric coherence maps obtained were linearly scaled from 0 (black) to 0.25 (white) and used to form the RGB color composite image shown in Fig. 16, where red corresponds to the $0.5 \times 0.5 \mathrm{~km}^{2}$ window, green to the $1 \times 1 \mathrm{~km}^{2}$ window, and blue for the $2 \times 2 \mathrm{~km}^{2}$ window.

A comparison with Fig. 13 makes clear that the coherence along the zone with a high differential FR is improved for all three cases. However, the color changes in Fig. 16 indicate that correction performance depends on the size of the estimation window. As noted in Section VI-A5, the optimum size of the window depends on the ionospheric conditions and the SNR 

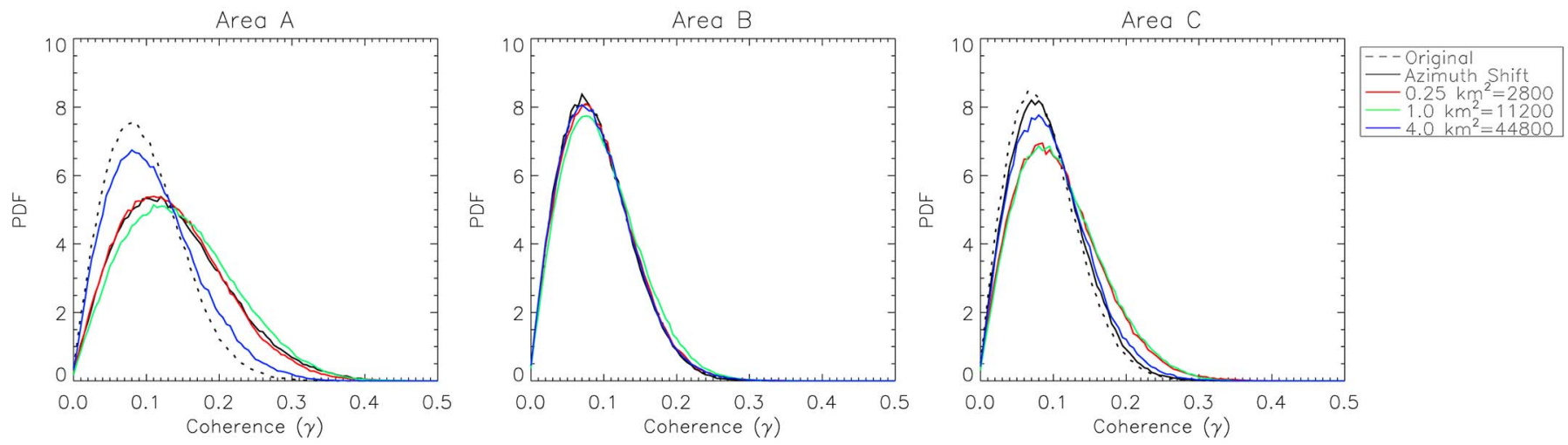

Fig. 17. Histograms of coherence values after scintillation correction for the A, B, and C areas in Figs. 15 and 16.

level, both of which vary within the scene. The yellowish tone in the high differential FR zone indicates that the smaller windows perform better, whereas in the parts of the scene with more homogeneous ionospheric conditions, the bluish horizontal stripes indicate, as expected, better performance of the larger window.

To better illuminate this, the performance of the correction in the rectangular areas marked A, B, and C in Figs. 15 and 16 is compared; all three areas are in the high differential FR zone. Fig. 17 shows the histograms of the original and corrected interferometric coherence values for the three areas. The dashed and solid black histograms indicate the original interferogram and after applying the conventional azimuth shift correction [20], respectively, whereas the red, green, and blue histograms correspond to scintillation corrections with window sizes of $0.5 \times 0.5 \mathrm{~km}^{2}, 1 \times 1 \mathrm{~km}^{2}$, and $2 \times 2 \mathrm{~km}^{2}$, respectively.

In area $\mathrm{A}$, the proposed correction performs slightly better than the simple azimuth shift correction, which aims only to compensate for the decorrelation due to residual azimuth shifts, not defocusing induced by scintillation. The best performance is obtained with the $1 \times 1 \mathrm{~km}^{2}$ window, and it degrades when the window size becomes too large, e.g., $2 \times 2 \mathrm{~km}^{2}$. In area B, none of the correction approaches improve the coherence, perhaps because the scintillation is too strong or the applied ionospheric altitude is incorrect. In area $\mathrm{C}$, the proposed scintillation correction performs better for the smaller window sizes $\left(0.5 \times 0.5 \mathrm{~km}^{2}\right.$ and $\left.1 \times 1 \mathrm{~km}^{2}\right)$.

Finally, the optimal size of the window is evaluated by means of a PSD analysis. Fig. 18 shows the PSD of the TEC estimates derived from FR for the master (red) and slave (green) acquisitions. The lower level of the slave PSD is consistent with the calmer ionospheric conditions during this acquisition. The regimes dominated by physics and noise (see Section IV-D) are clearly distinguishable. For the master acquisition, the critical irregularity wavenumber is around $1 /(800 \mathrm{~m})$ supporting the performance rank (better for small windows and worse for larger windows) shown in the previous paragraph.

\section{CONCLUSION}

This paper has shown that it is possible to correct scintillation effects on SAR images using estimates of FR under the thin ionospheric layer assumption. The performance of the correction

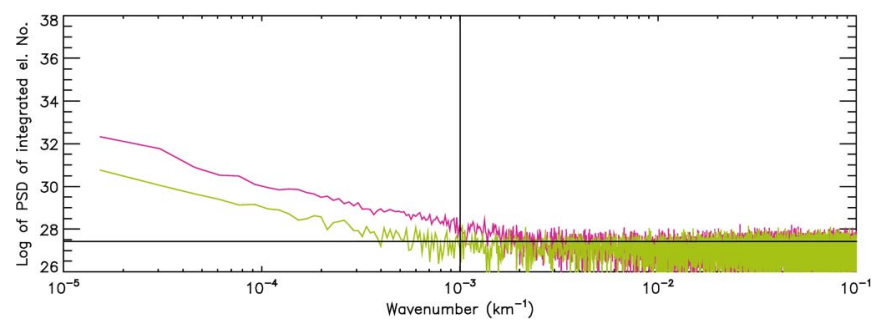

Fig. 18. PSD of TEC estimated from the ALOS data. The red line is estimated from the disturbed master acquisition and the green plot from the quite slave acquisition.

depends on ionospheric conditions, geomagnetic latitude, SNR, and the number of looks available to estimate FR. When correcting real data, the only controllable parameter is the number of looks associated to the used estimation window size, which can be optimized based on the frequency analysis of the spatial distribution of the TEC. The proposed correction methodology requires fully polarimetric data to estimate FR, and its performance is limited by the accuracy of the FR estimates.

The correction on partially focused SAR data at the effective altitude of thin ionospheric layer has been also proposed. Accordingly, both azimuth shifts (which lead to decorrelation in InSAR) and ionosphere scintillation can be compensated simultaneously. This idea is very general that, as far as the accurate TEC distribution is provided (regardless of how it is estimated), this correction method can correct any distortions on SAR data induced by the variation of the phase history within the synthetic aperture.

A method to estimate the effective altitude of the ionosphere is given in the Appendix. This is applicable not only to FR but also to azimuth shifts or any other kind of ionospheric distortions in SAR data, as far as they are detectable.

All information required for applying the correction (i.e., the TEC distribution, its spectra, and the ionospheric altitude) is derived from the SAR data themselves. Hence, this paper also highlights the potential of SAR as an instrument for quantitative monitoring of the ionosphere.

\section{APPENDIX}

The altitude of the ionosphere can be estimated by means of the parallax between azimuth sublooks. The ionosphere is 


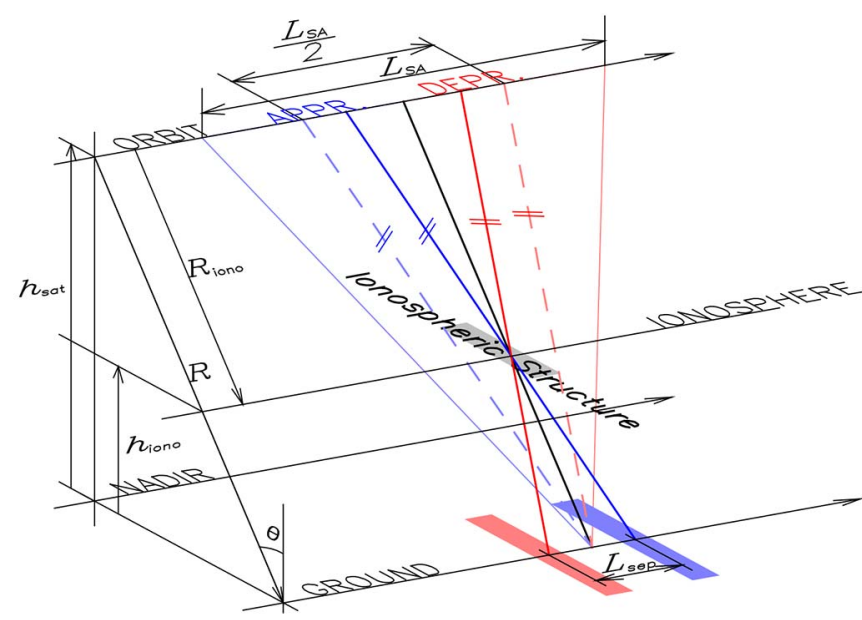

Fig. 19. Geometry of ionospheric altitude estimation.
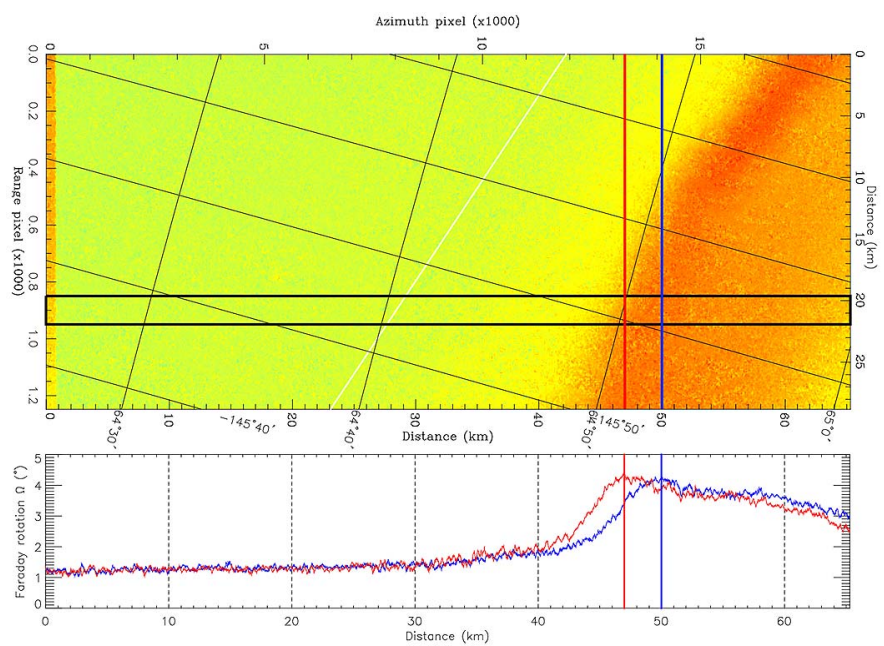

Fig. 20. (Top) Full-band estimation of FR and (bottom) subband estimation profiles along azimuth direction for (blue) approaching and (red) departing conditions.

located between the ground and the sensor; thus, when imaged, their distortions introduced in the SAR image (e.g., FR or azimuth shift) are shifted forward in the approaching subband and backward in the departing subband, as shown in Fig. 19. The altitude of the ionosphere is therefore proportional to the spatial separation of the detected ionospheric distortions in two azimuth subband images, i.e., $L_{\text {sep }}$

$$
h_{\text {iono }}=2 R_{0} \cos \theta \cdot \frac{L_{\mathrm{sep}}}{L_{\mathrm{SA}}}=2 h_{\mathrm{sat}} \frac{L_{\mathrm{sep}}}{L_{\mathrm{SA}}}
$$

where $\theta$ is the look angle.

Fig. 20 shows the estimation of $L_{\mathrm{sep}}$ in the ALOS Fairbanks data set using subband FR estimates. The synthetic aperture length is about $20 \mathrm{~km}$, the slant range is $770 \mathrm{~km}$ at the scene center, and the incidence angle is $24^{\circ}$. The estimated separation $L_{\text {sep }}$ is approximately $3 \mathrm{~km}$; hence, the estimated height of the ionosphere is $190 \mathrm{~km}$. There is still approximately $60 \mathrm{~km}$ of difference (see below) in the estimation of altitude. Nonetheless, the proposed method yields a better estimation of ionospheric

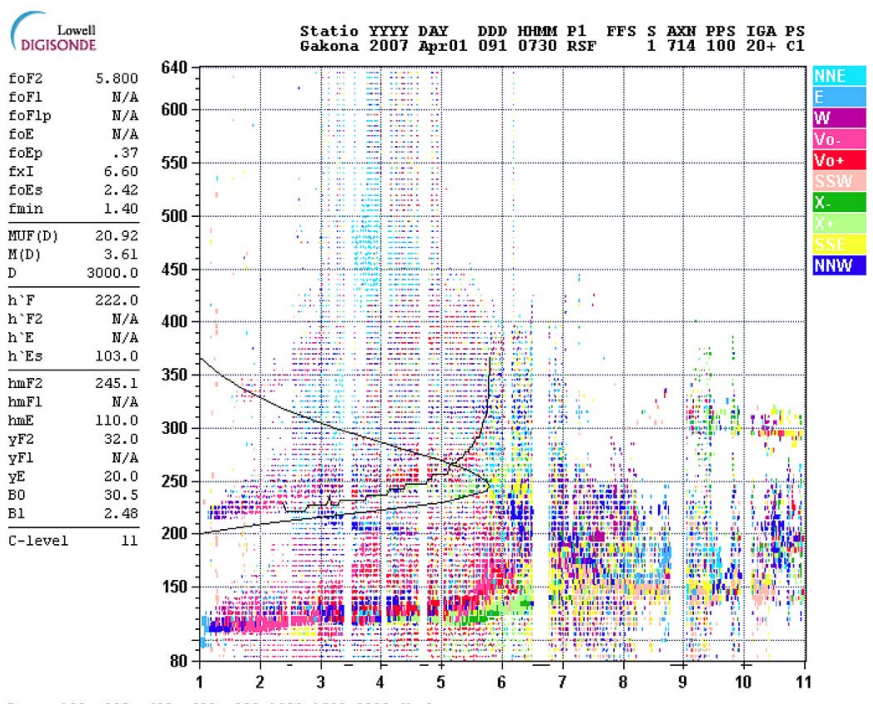

$\begin{array}{llllllllll}D & 100 & 200 & 400 & 600 & 800 & 1000 & 1.500 & 3000 & {[\mathrm{kmm}]} \\ \text { MUF } & 6.5 & 6.6 & 6.9 & 7.5 & 8.2 & 9.3 & 12.6 & 20.9 & {[\mathrm{HHz}]}\end{array}$

Fig. 21. Ionosonde observation at HAARP (around $200 \mathrm{~km}$ south of the scene center) at the time of master acquisition of Fairbanks data set [49].

altitude than simply assuming a fixed value of $350-400 \mathrm{~km}$. Fig. 21 shows ionosonde measurements at HAARP taken $200 \mathrm{~km}$ south of the scene center. [49], [50]. The $x$-axis of the plot is the plasma frequency in megahertz. Here, $1 \mathrm{MHz}$ corresponds to electron density of $1.24 \times 10^{10}$ electrons $/ \mathrm{m}^{3}$ [see (1)]. There are two solid black curves in the ionogram, which indicate the plasma frequencies, thus the electron densities, at that height. The upper exponential-like curve indicates the virtual height obtained by simple division of the travel time with $2 c_{0}$, whereas the lower rotated bell-shaped curve shows the true height estimated by considering the delay of group velocity in the ionosphere. (The image had been downloaded from [49], which is now obsolete. The same ionogram is currently available at [50] with different interpretations.) For more information for the interpretation of ionogram, we recommend [53] (in German).

The FR can be also calculated at the estimated ionospheric altitude as (19) does not depend on the focusing altitude. For SLCs affected by severe scintillations, such as the master ALOS Fairbanks acquisition, this helps preserve smaller scale variations of FR.

The estimation of the ionospheric altitude using azimuth parallax is only possible when the ionospheric feature shows a strong spatial variation. This means that the altitude of flat and plain ionosphere cannot be estimated using its parallax simply because there is no parallax. Nevertheless, as we have shown in Fig. 11 in Section VI-A6, altitude estimation is not a critical problem for slowly varying ionospheres.

\section{ACKNOWLEDGMENT}

The authors would like to thank JAXA and Prof. F. Meyer of the University of Alaska at Fairbanks, Fairbanks, AK, USA, for providing the ALOS PALSAR data takes and N. C. Rogers of The University of Sheffield, Sheffield, U.K., for providing the ionospheric phase screens generated by the WBMOD. 


\section{REFERENCES}

[1] IEEE Standard Definitions of Terms for Radio Wave Propagation, IEEE Standards Board, New York, NY, USA, 1997.

[2] K. Davies, Ionospheric Radio Waves. London, U.K.: Peregrinus, 1990.

[3] D. P. Belcher, "Theoretical limits on SAR imposed by the ionosphere," IET Radar, Sonar Navig., vol. 2, no. 6, pp. 435-448, Dec. 2008.

[4] J. Aaron, "Global morphology of ionospheric scintillation," Proc. IEEE, vol. 70, no. 4, pp. 360-378, Apr. 1982.

[5] F. J. Meyer, R. Bamler, N. Jakowski, and T. Fritz, "The potential of lowfrequency SAR systems for mapping ionospheric TEC distributions," IEEE Geosci. Remote Sens. Lett., vol. 3, no. 4, pp. 560-564, Oct. 2006

[6] A. L. Gray, K. E. Mattar, and G. Sofko, "Influence of ionospheric electron density fluctuations on satellite radar interferometry," Geophys. Res. Lett., vol. 27, no. 10, pp. 1451-1454, May 2000.

[7] U. Wegmüller, C. Werner, T. Strozzi, and A. Wiesmann, "Ionospheric electron concentration effects on SAR and INSAR," in Proc. IEEE IGARSS, Denver, CO, USA, 2006, pp. 3731-3734.

[8] K. C. Yeh and C. H. Liu, "Waves in fluid plasma with a steady magnetic field," in Ionospheric Radio Waves, 1st ed. San Diego, CA, USA: Academic, 1972, p. 192.

[9] J. S. Kim, "Development of ionosphere estimation techniques for the correction of SAR data," Ph.D. dissertation, Dept. Civil, Environ. Geomatic Eng., ETH, Zürich, Switzerland, 2013.

[10] F. J. Meyer and J. B. Nicoll, "Prediction, detection and correction of Faraday rotation in full-polarimetric L-band SAR data," IEEE Trans. Geosci. Remote Sens., vol. 46, no. 10, pp. 3076-3086, Oct. 2008.

[11] Z.-W. Xu, J. Wu, and Z.-S. Wu, "A survey of ionospheric effects on space-based radar," Waves Random Media, vol. 14, no. 2, pp. S189-S273, Apr. 2004

[12] F. Meyer, "Performance requirements for correction of ionospheric signals in L-band SAR data," in Proc. EUSAR, Aachen, Germany, 2010, pp. 1-4.

[13] F. J. Meyer, "Performance requirements for ionospheric correction of lowfrequency SAR data," IEEE Trans. Geosci. Remote Sens., vol. 49, no. 10, pp. 3694-3702, Oct. 2011

[14] European Space Agency, "Biomass: Report for mission selection," ESA Communication Production Office, Noordwijk, The Netherlands, SP-1324/1, 2012. Available: https://earth.esa.int/web/guest/documentlibrary/browse-document-library/-/article/biomass-report-for-missionselection-an-earth-explorer-to-observe-forest-biomass

[15] M. Shimada, M. Watanabe, T. Motooka, M. Ohki, and Y. Wada, "PALSAR-2 and Pi-SAR-L2-Multi frequency polarimetric sensitivity on disaster," in Proc. EUSAR, Berlin, Germany, 2014, pp. 1-2.

[16] N. Gebert, B. G. Domínguez, M. W. J. Davidson, M. D. Marin, and P. Silvestrin, "SAOCOM-CS-A passive companion to SAOCOM for single-pass L-band interferometry," in Proc. EUSAR, Berlin, Germany, 2014, pp. $1-4$.

[17] S. Hensley et al., "Science performance of the NASA-ISRO spaceborne SAR mission concept," in Proc. IEEE IGARSS, Québec, QC, Canada, 2014.

[18] I. Hajnsek et al., "Tandem-L: Science requirements and mission concept," in Proc. EUSAR, Berlin, Germany, 2014, pp. 1-4

[19] N. Rogers, S. Quegan, J. S. Kim, and K. Papathanassiou, "Impacts of ionospheric scintillation on the BIOMASS P-band satellite SAR," IEEE Trans. Geosci. Remote Sens., vol. 52, no. 3, pp. 1856-1868, Mar. 2014.

[20] J. Chen and H. A. Zebker, "Ionospheric artifacts in simultaneous L-band InSAR and GPS observations," IEEE Trans. Geosci. Remote Sens. vol. 50, no. 4, pp. 1227-1239, Apr. 2012.

[21] A. C. Chen and H. A. Zebker, "Reducing ionospheric effects in InSAR data using accurate coregistration," IEEE Trans. Geosci. Remote Sens. vol. 52, no. 1, pp. 60-70, Jan. 2014.

[22] J. S. Kim, A. Danklmayer, and K. Papathanassiou, "Correction of ionospheric distortions in low frequency interferometric SAR data," in Proc. IEEE IGARSS, Vancouver, BC, Canada, 2011, pp. 1505-1508,

[23] R. S. Conker, M. B. El-Arini, C. J. Hegarty, and T. Hsiao, "Modeling the effects of ionospheric scintillation on GPS/satellite-based augmentation system availability," Radio Sci., vol. 38, no. 1, pp. 1:1-1:23, Feb. 2003.

[24] A. Ishimaru, Y. Kuga, J. Liu, Y. Kim, and T. Freeman, "Ionospheric effects on synthetic aperture radar at $100 \mathrm{MHz}$ to $2 \mathrm{GHz}$," Radio Sci., vol. 34, no. 1, pp. 257-268, Jan./Feb. 1999.

[25] R. Z. Schneider and K. Papathanassiou, "Estimation and correction of ionospheric induced phase errors in SAR images using coherent scatterers," in Proc. IEEE IGARSS, Cape Town, South Africa, 2009, pp. IV-165-IV-168.

[26] M. Shimada, Y. Muraki, and Y. Otsuka, "Discovery of anomalous stripes over the Amazon by the PALSAR onboard ALOS satellite," in Proc. IEEE IGARSS, Boston, MA, USA, 2008, pp. II-387-II-390.
[27] C. S. Carrano, K. M. Groves, and R. G. Caton, "Simulating the impacts of ionospheric scintillation on L-band SAR image formation," Radio Sci. vol. 47, no. 4, Aug. 2012, Art. ID. RSOL20.

[28] E. V. Appleton, "Wireless studies of the ionosphere," J. Inst. Electr. Eng., vol. 71, no. 430, pp. 642-650, 1932.

[29] S. Schaer, W. Gurtner, and J. Feltens, "IONEX: The IONosphere map exchange format version 1," in Proc. IGS AC Workshop, Darmstadt, Germany, 1998, pp. 233-247.

[30] S. McLean, S. Macmillan, S. Maus, V. Lesur, A. Thomson, and D. Dater, "The US/UK World Magnetic Model for 2005-2010," Nat. Ocean. Atmos. Admin., Washington, DC, USA, NOAA Tech. Rep. NESDIS/NGDC-1, 2004

[31] D. P. Belcher and N. C. Roger, "Theory and simulation of ionospheric effects on synthetic aperture radar," IET Radar, Sonar Navig., vol. 3 , no. 5, pp. 541-551, Oct. 2009.

[32] R. E. Robins, J. A. Secan, and E. J. Fremouw, "A mid-latitude scintillation model," Northwest Res. Assoc., Redmond, WA, USA, Tech. Rep. NWRA-86-R0004, 1986.

[33] F. Meyer, "Quantifying ionosphere-induced image distortions in L-band SAR data using the ionospheric scintillation model WBMOD," in Proc. EUSAR, Berlin, Germany, 2014, pp. 1-4.

[34] S. R. Cloude, Polarisation: Applications in Remote Sensing. New York, NY, USA: Oxford Univ. Press, 2010.

[35] A. Freeman and S. Saachi, "On the detection of Faraday rotation in linearly polarized L-band SAR backscatter signatures," IEEE Trans. Geosci. Remote Sens., vol. 42, no. 8, pp. 1607-1616, Aug. 2004

[36] J. S. Kim and K. Papathanassiou, "Faraday rotation estimation performance analysis," in Proc. EUSAR, Aachen, Germany, 2010, pp. 182-185.

[37] S. H. Bickel and R. T. H. Bates, "Effects of magneto-ionic propagation on the polarization scattering matrix," Proc. IEEE, vol. 53, no. 8 , pp. 1089-1901, Aug. 1965

[38] A. Freeman, "Calibration of linearly polarized polarimetric SAR data subject to Faraday rotation," IEEE Trans. Geosci. Remote Sens., vol. 42 , no. 8, pp. 1617-1624, Aug. 2004

[39] J. Chen and S. Quegan, "Improved estimators of Faraday rotation in spaceborne polarimetric SAR data," IEEE Geosci. Remote Sens. Lett., vol. 7, no. 4, pp. 846-850, Oct. 2010.

[40] S. Quegan et al., "Ionospheric mitigation schemes and their consequences for BIOMASS product quality," Eur. Space Agency, Paris, France, 2012.

[41] N. C. Rogers and S. Quegan, "The accuracy of Faraday rotation estimation in satellite system aperture radar images," IEEE Trans. Geosci. Remote Sens., vol. 52, no. 8, pp. 4799-4807, Aug. 2014.

[42] R. F. Hansen, Radar Interferometry. Dordrecht, The Netherlands, Kluwer, 2001

[43] R. Bamler and P. Hartl, "Synthetic aperture radar interferometry," Inv. Probl., vol. 14, no. 4, pp. R1-R54, Aug. 1998.

[44] E. Rodriguez and J. M. Martin, "Theory and design of interferometric synthetic aperture radars," Proc. Inst. Elect. Eng.-F Radar Signal Process. vol. 139, no. 2, pp. 147-159, Apr. 1992.

[45] J. S. Kim, K. P. Papathanassiou, S. Quegan, and N. Rogers, "Estimation and correction of scintillation effects on spaceborne P-band SAR images," in Proc. IEEE IGARSS, Munich, Germany, 2012, pp. 5101-5104.

[46] J. C. Curlander and R. N. McDonough, Synthetic Aperture Radar: System and Signal Processing. New York, NY, USA: Wiley, 1991.

[47] R. Scheiber, S.-K. Lee, K. P. Papathanassiou, and N. Floury, "Extrapolation of airborne polarimetric and interferometric SAR data for validation of bio-geo-retrieval algorithms for future spaceborne SAR missions," in Proc. IEEE IGARSS, Cape Town, South Africa, 2009, pp. II-941-II-944.

[48] J. A. Secan, WBMOD Ionospheric Scintillation Model-An Abbreviated User's Guide (v. 15). Tucson, AZ, USA: North West Research Institute, Aug. 2007.

[49] "The High Frequency Active Auroral Research Program," [Accessed 27 August 2010]. [Online]. Available: http://maestro.haarp.alaska.edu/cgibin/ashtech/tec.cgi?type $=$ ut \&year $=2007 \&$ month $=4 \&$ day $=1$

[50] "GIRO Database," [Accessed 103 2015]. [Online]. Available: http:// car.uml.edu/common/ShowIonogramPage?mid $=8313471 \&$ ursiCode $=$ GA762\& time $=2007.04 .01 \% 20(091) \% 2007: 30: 00.000$

[51] K. P. Papathanassiou and S. R. Cloude, "Single-baseline polarimetric SAR interferometry," IEEE Trans. Geosci. Remote Sens., vol. 39, no. 11, pp. 2352-2363, Nov. 2001.

[52] D. B. Gesch, "The national elevation dataset," in Digital Elevation Model Technologies and Applications-the DEM Users Manual, 2nd ed., D. Maune, Ed. Bethesda, MD, USA: ASPRS, pp. 99-118, 2007

[53] J. Mielich, "Über die Lesbarkeit von Digisonden-Ionogrammen," Jun. 2012. [Accessed 113 2015]. [Online]. Available: https://www.darc.de/ uploads/media/Digisonde-Ionogramm-Erk1\%C3\%A4rung.pdf 


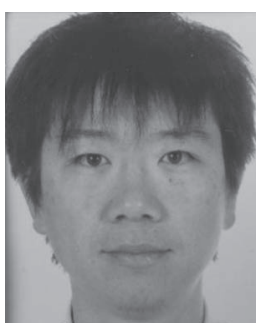

Jun Su Kim was born in Busan, Korea, in 1982. He received the Bachelor's and Master's degrees in earth environmental sciences from Seoul National University, Seoul, Korea, in 2004 and 2007, respectively, and the Ph.D. degree from the Swiss Federal Institute of Technology in Zurich (ETH Zurich), Zurich, Switzerland, in 2014.

Since 2008, he has been with the Microwaves and Radar Institute, German Aerospace Center (DLRHR), Wessling, Germany.

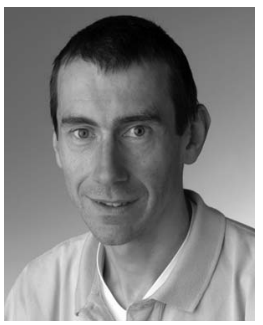

Konstantinos P. Papathanassiou (AM'01-M'06SM'09-F'13) received the Dipl.Ing. (Hons.) and Dr. (Hons.) degrees from the Graz University of Technology, Graz, Austria, in 1994 and 1999, respectively.

From 1992 to 1994, he was with the Institute for Digital Image Processing (DIBAG), Joanneum Research, Graz. Between 1995 and 1999, he was with the Microwaves and Radar Institute, German Aerospace Center (DLR-HR), Wessling, Germany. From 1999 to 2000, he was a European Union Postdoctoral Fellow with Applied Electromagnetics, St. Andrews, U.K. Since October 2000, he has been a Senior Scientist with DLR-HR, leading the Information Retrieval Research Group. He is the author or coauthor of more than 100 publications in international journals, conferences, and workshops. His main research interests are in polarimetric and interferometric processing and calibration techniques, polarimetric SAR interferometry, and the quantitative parameter estimation from SAR data, as well as in SAR mission design and SAR mission performance analysis.

Dr. Papathanassiou was the recipient of the IEEE GRSS IGARSS Symposium Prize Paper Award in 1998, the Best Paper Award of the European SAR Conference in 2002, the DLR Science Award in 2002, and the DLR Senior Scientist Award in 2011.

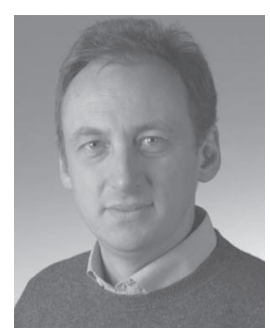

Rolf Scheiber received the Diploma in electrical engineering from the Technical University of Munich, Munich, Germany, in 1994 and the Ph.D. degree in electrical engineering from the Karlsruhe Institute of Technology, Karlsruhe, Germany, in 2003, with a thesis on airborne SAR interferometry.

Since 1994, he has been with the Microwaves and Radar Institute, German Aerospace Center (DLRHR), Wessling, Germany, where he developed the operational high-precision interferometric SAR processor for its experimental SAR airborne sensor. Since 2001, he has been the Head of the SAR Signal Processing Group, Department of SAR Technology, DLR-HR. His research interests include algorithm development for high-resolution airborne and spaceborne SAR focusing, SAR interferometry, differential SAR interferometry, and SAR tomography, as well as radio sounding algorithms and applications.

Dr. Scheiber was a corecipient of the IEEE Geoscience and Remote Sensing Society Transactions Prize Paper Award in 1996 for the contribution "Extended Chirp Scaling Algorithm for Air and Spaceborne SAR Data Processing in Stripmap and ScanSAR Imaging Modes."

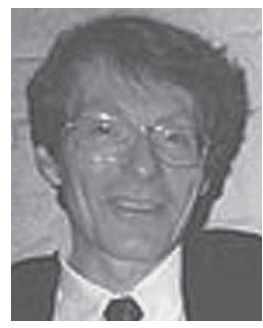

Shaun Quegan (M'90) received the B.A. and M.Sc. degrees in mathematics from the University of Warwick, Coventry, U.K., in 1970 and 1972, respectively, and the Ph.D. degree from The University of Sheffield, Sheffield, U.K., in 1982.

From 1982 to 1986, he was a Research Scientist with the Marconi Research Centre, Great Baddow, U.K., and led the Remote Sensing Applications Group from 1984 to 1986 . He established the SAR Research Group at The University of Sheffield in 1986, whose success led to his professorship awarded in 1993. In the same year, he helped inaugurate the Sheffield Centre for Earth Observation Science, of which he remains the Director. In 2001 , he became the Director of the Centre for Terrestrial Carbon Dynamics, U.K. National Environmental Research Council. This multi-institutional center is concerned with assimilating earth observation and other data into process models of the land component of the carbon cycle and now forms part of the U.K. National Centre for Earth Observation. His broad interests in the physics, systems, and data analysis aspects of radar remote sensing are now subsumed in the more general aim of exploiting many sorts of electrooptic technology to give greater quantitative understanding of the carbon cycle. 